\title{
On the self-enrichment scenario of galactic globular clusters: constraints on the IMF
}

\author{
N. Prantzos ${ }^{1}$ and C. Charbonnel ${ }^{2,3}$ \\ 1 Institut d'Astrophysique de Paris, UMR7095 CNRS, Univ. P. \& M. Curie, 98bis Bd. Arago, 75104 Paris, France \\ e-mail: prantzos@iap.fr \\ 2 Geneva Observatory, 1290 Sauverny, Switzerland \\ e-mail: Corinne.Charbonnel@obs . unige.ch \\ 3 Laboratoire d'Astrophysique de Toulouse et Tarbes - CNRS UMR 5572 CNRS - Université Paul Sabatier Toulouse 3, 14, \\ Av. E. Belin, 31400 Toulouse, France
}

Received 6 April 2006 / Accepted 22 June 2006

ABSTRACT

\begin{abstract}
Aims. Galactic globular cluster (GC) stars exhibit abundance patterns that are not shared by their field counterparts, e.g. the welldocumented $\mathrm{O}-\mathrm{Na}$ and $\mathrm{Mg}-\mathrm{Al}$ anticorrelations. Recent spectroscopic observations of GC turnoff stars have provided compelling evidence that these abundance anomalies were already present in the gas from which the observed stars formed. A widely held hypothesis is that the gas was "polluted" by stars that were more massive (and evolving faster) than the presently observed low-mass stars. In the framework of this "self-enrichment" scenario for GCs, we present a new method of deriving the initial mass function (IMF) of the polluters, by using the $\mathrm{O} / \mathrm{Na}$ abundance distribution.

Methods. We focus on NGC 2808, a GC for which the largest sample of O and Na abundance determinations is presently available. We use the abundance distribution of $[\mathrm{O} / \mathrm{Na}]$ to derive the amount of polluted material with respect to the original composition. We explore two scenarios in detail for the self-enrichment of the cluster, which differ by the assumptions made on the composition of the polluter ejecta. In each case we consider two classes of possible "culprits": massive asymptotic giant branch (AGB) stars (4-9 $\left.M_{\odot}\right)$ and winds of massive stars (WMS) in the mass range 10-100 $M_{\odot}$.

Results. We obtain upper limits for the slope of the IMF (assumed to be given by a power-law) of the stars initially more massive than the present turnoff mass. We also derive lower limits for the amount of stellar residues in NGC 2808.

Conclusions. We find that the polluter IMF had to be much flatter than the presently observed IMFs in stellar clusters, which agrees with the results of two other GC IMF determination methods, which we also discuss. Likewise, we find that the present mass of the GC should be totally dominated by stellar remnants if the polluters were AGB stars, which is not the case if the culprits are WMS. We critically analyse the advantages and shortcomings of each potential polluter class and find the WMS scenario more attractive.
\end{abstract}

Key words. stars: abundances - stars: luminosity function, mass function - Galaxy: abundances - globular clusters: general globular clusters: individual: NGC 2808

\section{Abundance anomalies in galactic globular clusters and the self-enrichment scenario}

Globular cluster (GC) studies are linked to a wide variety of astrophysical issues. These stellar systems indeed provide insight into the formation and early chemical evolution of galaxies and of their substructures. They also represent unique laboratories for dynamical processes that occur on shorter timescales than Hubble time (such as two-body relaxation, mass segregation from equipartition of energy, stellar encounters, collisions and mergers, core collapse, tidal stripping). Because they are large aggregates of coeval stars, all located at the same distance from us and containing both very common and unusual (e.g., blue stragglers) stellar objects, they provide invaluable tests of stellar evolution theory. However, and despite their broad astrophysical importance, their origin and formation processes still remain uncertain.

For all these reasons, galactic GCs have been subject to intensive scrunity over the past decades. The advent of very large telescopes and of multiple-object spectrometers allowed detailed investigation of their chemical composition (see Gratton et al. 2004; and Sneden 2005, for recent and extended reviews). We briefly recall here the fundamental chemical properties of the galactic GCs:

(i) most of them appear to be mono-metallic as far as the Fe-group elements $(\mathrm{Fe}, \mathrm{Ni}, \mathrm{Cu}$ ) are concerned (with the notable exception of Omega Cen);

(ii) they present very low scatter and the same trends as field stars for the neutron-capture elements (both s- and r-; Ba, $\mathrm{La}, \mathrm{Eu})$ and the alpha-elements ( $\mathrm{Si}, \mathrm{Ca})$;

(iii) for the lighter elements (from $\mathrm{C}$ to $\mathrm{Al}$ ), they exhibit complex patterns and large star-to-star abundance variations, which are not shared by their field counterparts. Among these anomalous patterns, the most striking ones are the anticorrelations between the abundances of $\mathrm{O}$ and $\mathrm{Na}$ (see Pilachowski 1989; Drake et al. 1992; and Sneden et al. 1991, for the early studies; for complete references see Gratton et al. 2004) and of $\mathrm{Mg}$ and $\mathrm{Al}$ (see e.g. Shetrone 1996; Ivans et al. 1999; Ramirez \& Cohen 2002) ${ }^{1}$.

${ }^{1}$ Star-to-star variations of the strengths of the lines of these elements ( $\mathrm{Na}$ and $\mathrm{Al}$ in particular) were known to exist at the tip of the red giant branch (RGB) for a long time (Cohen 1978; Peterson 1980; Norris et al. 1981; see Kraft 1994, for an early review). 
These anticorrelations are easily understood as the result of proton-capture nucleosynthesis in the $\mathrm{CNO}$-cycle and the $\mathrm{NeNa}$ and MgAl-chains of H-burning (Denissenkov \& Denissenkova 1990; Langer et al. 1993). Proton-captures on ${ }^{16} \mathrm{O}$ and ${ }^{22} \mathrm{Ne}$ respectively lead to the destruction of $\mathrm{O}$ and to the production of ${ }^{23} \mathrm{Na}$ at temperatures $>2 \times 10^{7} \mathrm{~K}$. At higher temperatures $(\sim 4 \times$ $\left.10^{7} \mathrm{~K}\right),{ }^{20} \mathrm{Ne}$ generates ${ }^{23} \mathrm{Na}$. On the other hand, the MgAl-chain is only active at temperatures higher than $\sim 7 \times 10^{7} \mathrm{~K}$.

Although the underlying nucleosynthesis is unambiguous, the identification of the corresponding astrophysical site still remains a challenge. For a long time the main question was whether such patterns were inherited at the birth of the stars that we are currently observing (the so-called self-enrichment hypothesis that implies pollution of the intracluster gas by more massive and faster evolving stars) or if they were generated in the course of the evolution of these objects (the so-called evolution hypothesis that requires non-canonical mixing inside the star itself). A major breakthrough was made in that respect from highresolution spectrographs on very large telescopes that allowed one to determine the abundances of the concerned elements in faint subgiant and turnoff stars in a couple of GCs (Gratton et al. 2001; Grundhal et al. 2002; Carretta et al. 2003, 2004; Ramirez $\&$ Cohen 2002, 2003). The discovery of the above-mentioned anticorrelations in these scarcely evolved objects has given a new spin to the self-enrichment scenario. Indeed, turnoff stars are not hot enough for the required nuclear reactions to occur in their interiors. Additionally, the spread of abundances cannot be explained by in situ mechanisms like gravitational settling and thermal diffusion (Richard et al. 2002; Michaud et al. 2004). This implies that the $\mathrm{O}, \mathrm{Na}, \mathrm{Mg}$, and $\mathrm{Al}$ abundance anomalies pre-existed in the material out of which these objects formed; their gas must have been polluted early in the history of the cluster by more massive and faster evolving stars (Cottrell \& Da Costa 1981) $)^{2}$.

It has long been debated whether GCs were made out of totally metal-free material or out of the same gas as the halo field stars (progressively metal-enriched, during the halo chemical evolution). The former is the "classical" self-enrichment scenario (Cayrel 1986; Fall \& Rees 1985; Truran et al. 1991; Brown et al. 1991; Parmentier et al. 1999; Parmentier 2004), while the latter is a "pre-enrichment" scenario (e.g. Harris \& Pudritz 1994).

The classical self-enrichment scenario assumes that stars within the GCs created the totality of the metals (light and heavy), and it involves three stellar generations. A first generation of exclusively massive stars is clustered in the central regions of the progenitor cloud, and their SNII explosions rapidly enrich the intra-cluster gas in iron, $\alpha$-, and heavy elements, but not in s-elements; the resulting $[\mathrm{Ba} / \mathrm{Eu}]$ and $[\mathrm{Sr} / \mathrm{Ba}]$ ratios should thus reflect pure r-process synthesis (Truran 1988). A second stellar generation is formed out of this gas, and a subclass of rapidly evolving stars injects into the ISM products of H-burning (and nothing else). The third generation formed from these ejecta has the same uniform heavy-element content (in

\footnotetext{
2 An alternative would be that the abundance anomalies were accreted onto the surface of low-mass stars from the mass loss by massive stars (D'Antona et al. 1983; Thoul et al. 2002). In this case, only the surface convective layers would have a polluted composition, and the anomalies would disappear on the RGB due to dilution during the first dredge-up event. However, turnoff and bright giant stars exhibit the same anti-correlations, which means that the entire star, or at least $80 \%$ of its mass (which corresponds approximately to the maximum depth of the convective envelope during the first dredge-up), has the composition of the photosphere.
}

alpha- and heavier than Fe elements) as the second one, but a dispersion in its light metal abundances. The long-lived stars of the second and third generations are those observed today.

On the other hand, the pre-enrichment scenario assumes that GCs were born with their current heavy metal content and invokes only two stellar generations: the first of them produces only the light metals, which contaminate the gas of the second generation, and builds up the observed anticorrelations (i.e. they are equivalent to the second and third generations of the previous scenario).

The two scenarios differ on a key point, the slow neutron capture component of the heavy elements (s-component). In the first scenario, only the rapid neutron capture component (r-component), produced in SNII, may exist, whereas in the latter the s-component appears naturally (exactly as in the field stars of similar metallicity). A recent high-resolution and high$\mathrm{S} / \mathrm{N}$ spectroscopic analysis of turnoff and early subgiant stars in three GCs spanning a wide range in $[\mathrm{Fe} / \mathrm{H}]$ values (James et al. 2004a,b) showed that the $[\mathrm{Ba} / \mathrm{Eu}]$ and $[\mathrm{Sr} / \mathrm{Ba}]$ ratios in $\mathrm{GCs}$ follow the trends found in field stars over the same metallicity range. Since $\mathrm{Ba}$ and $\mathrm{Sr}$ are s-elements, while $\mathrm{Eu}$ is an r-element, these findings clearly indicate a similar and uniform enrichement by both $r$ - and s-process nucleosynthesis in both populations (halo field stars and GCs). Consequently, the "classical" self-enrichment scenario is disfavoured.

Another important result of the work of James et al. (2004a,b) is that no dispersion is found for s-element abundances within each individual GC (see also Armosky et al. 1994), nor any correlation with the $\mathrm{O}$ and $\mathrm{Na}$ patterns. This indicates that the stars responsible for the light element anticorrelations did not produce significant amounts of s-process elements.

In view of the aforementioned observational constraints, one concludes that the GC heavy metals must come from preenrichment and that only light metals up to Al are produced in situ. It is this scenario that will be called hereafter "the selfenrichment scenario".

One key aspect of the problem is that the O-Na anticorrelation is found in all GCs where it has been looked for, independent of their characteristics, i.e., in disk and halo GCs spanning a wide range in metallicity and in physical properties (such as horizontal branch morphology, total cluster mass, concentration, and density; see Carretta 2006; and Carretta et al. 2006). It appears to be an intrinsic property of those stellar systems. Understanding the related self-enrichment process would obviously shed light on GC formation and on the physical processes that dominate the primordial phases of their evolution.

In particular, one may try to constrain the initial mass function (IMF) of GCs in the framework of the self-enrichment scenario. Such an attempt was first made by Smith \& Norris (1982) on the basis of the then available data on $\mathrm{C}-\mathrm{N}$ anticorrelation in NGC 6752 and 47 Tuc. They found that the observed depletion of $\mathrm{C}$ in the cyanogen enhanced stars of those clusters can be achieved only if the IMF of the first generation stars had a slope $X<-0.4$ (where $X=1.35$ is the Salpeter value) and an upper mass limit $<11 M_{\odot}$.

In the present work we discuss the case of NGC 2808 as it is the GC for which the largest sample of $\mathrm{O}$ and $\mathrm{Na}$ abundance determinations is available. On the basis of recent data, we derive the $\mathrm{O} / \mathrm{Na}$ abundance distribution of the cluster (Sect. 2). We explore two scenarios for the self-enrichment (Sect. 3) and, in both cases, consider two possible classes of "polluters": (i) massive AGB stars (4 to $9 M_{\odot}$ ) which undergo hot-bottom burning and which are usually considered as the most likely culprits; and (ii) winds of rotating massive stars (WMS), which offer an 
interesting alternative, as we argue in Sect. 5. From the analysis of NGC 2808 data, we constrain the IMF of the stars that are initially more massive than the ones surviving today, i.e., the GC IMF above $0.8 M_{\odot}$, and we discuss the implications for the amount of stellar residues (Sect. 4). In (Sect. 5) we discuss the various (often extreme) assumptions made in the course of our analysis and argue that, in a realistic scenario, the IMF should be even flatter than the ones we derive in Sect. 4. We also discuss the advantages and shortcomings of the two classes of polluters, AGBs and WMS. In Sect. 6 we summarize our work.

\section{Abundance distribution of $[\mathrm{O} / \mathrm{Na}]$ and self-enrichment in NGC 2808}

In the case of NGC 2808, the O-Na anticorrelation was established for the first time by Carretta et al. (2004). In an extended survey, Carretta et al. (2006) detected both $\mathrm{Na}$ and $\mathrm{O}$ in 91 RGB stars of the cluster and obtained upper limits for $\mathrm{O}$ abundances for another 36 giants with $\mathrm{Na}$ abundance determinations. The spread in $\mathrm{O}$ and $\mathrm{Na}$ abundances is seen in stars of all luminosities along the RGB, pointing out a primordial origin with no significant contribution from the internal evolution of the stars (see also Carretta et al. 2003).

The data of Carretta et al. (2006) for the 91 stars where both $\mathrm{O}$ and $\mathrm{Na}$ are detected are displayed in Fig. 1. It is clearly seen that the ratio $[\mathrm{O} / \mathrm{Fe}]^{3}$ varies from $\sim 0.5$ down to $\sim-1$, i.e. by a factor of $\sim 30$, while at the same time $\mathrm{Na}$ increases from $[\mathrm{Na} / \mathrm{Fe}] \sim-0.2$ to $\sim 0.8$, i.e. by a factor of $\sim 10$. Regarding the O-depletion levels, NGC 2808 is very similar to M13 (Sneden et al. 2004); both clusters display the severest variations reported up to now. Since the value of $[\mathrm{O} / \mathrm{Fe}]=0.5$ is typical of field halo stars (e.g. Spite et al. 2005), it is usually assumed that stars displaying that ratio (and correspondingly low $[\mathrm{Na} / \mathrm{Fe}]$ ) are those that maintain their original composition, the one of the protocluster gas. In contrast, stars displaying the lowest $[\mathrm{O} / \mathrm{Fe}]$ and highest $[\mathrm{Na} / \mathrm{Fe}]$ have been severely contaminated by some external agent (in the framework of the self-enrichment scenario). Stars with intermediate compositions are born from gas that was contaminated to a lesser extent.

Because of the anticorrelation, the ratio $[\mathrm{O} / \mathrm{Na}]$ varies more than either $[\mathrm{O} / \mathrm{Fe}]$ or $[\mathrm{Na} / \mathrm{Fe}]$, from -1.55 to 0.55 , and offers a larger basis for statistical analysis of the abundance distribution within NGC 2808. Such a distribution has already been presented in Carretta et al. (2006), but we adopt a different (and, in our opinion, more appropriate) one here, obtained along the following lines:

The average error bars for $[\mathrm{O} / \mathrm{Fe}]$ and $[\mathrm{Na} / \mathrm{Fe}]$ in the $91-$ star sample of Carretta et al. (2006) are $\bar{\sigma}_{\mathrm{O}}=0.077 \mathrm{dex}$ and $\bar{\sigma}_{\mathrm{Na}}=0.098 \mathrm{dex}$, respectively. For each star, the error in the $[\mathrm{O} / \mathrm{Na}]$ ratio $\sigma_{\mathrm{ONa}}=\sqrt{\sigma_{\mathrm{O}}^{2}+\sigma_{\mathrm{Na}}^{2}}$ is calculated, and the average is found to be $\bar{\sigma}_{\mathrm{ONa}}=0.14$. We then consider bins in $[\mathrm{O} / \mathrm{Na}]$ of width $\Delta[\mathrm{O} / \mathrm{Na}]=0.30$, i.e. slightly larger than twice the average error on that ratio. Provided the quoted error bars reflect statistical errors reliably, it can be reasonably argued that stars found in different bins have a truly different composition (in a statistically significant way), at least as far as $\mathrm{O}$ and $\mathrm{Na}$ are concerned. The full range of the $[\mathrm{O} / \mathrm{Na}]$ spectrum is covered by seven such bins, with values of $[\mathrm{O} / \mathrm{Na}]$ ranging from -1.4 to 0.4 . The

\footnotetext{
${ }^{3}$ In the usual notation of spectroscopists, $[\mathrm{O} / \mathrm{Fe}]=\log _{10}(\mathrm{O} / \mathrm{Fe})_{*}-$ $\log _{10}(\mathrm{O} / \mathrm{Fe})_{\odot}$, where $*$ and $\odot$ denote stellar and solar values, respectively.
}

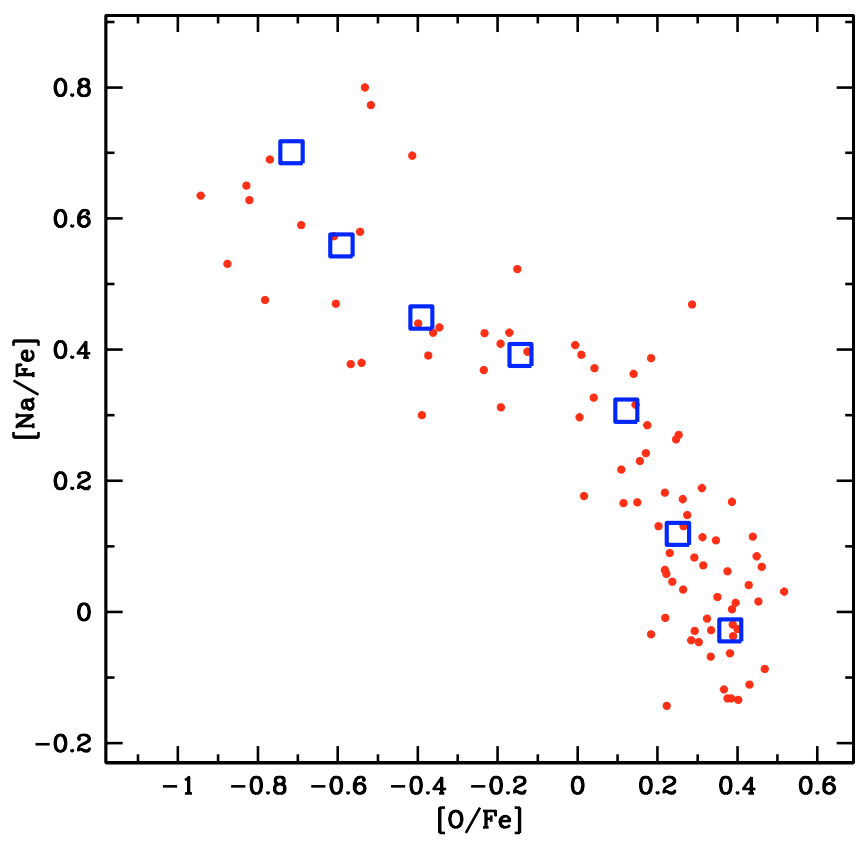

Fig. 1. Na vs. O anticorrelation in NGC 2808. Data for a sample of 91 stars, with both $\mathrm{Na}$ and $\mathrm{O}$ detections, are from Carretta et al. (2006). Open squares indicate average abundances in the seven bins of the adopted $\mathrm{O} / \mathrm{Na}$ distribution (see Fig. 2 and Sect. 2).

average abundances of $[\mathrm{O} / \mathrm{Fe}]$ and $[\mathrm{Na} / \mathrm{Fe}]$ in the seven $[\mathrm{O} / \mathrm{Na}]$ bins are shown in Fig. 1.

The resulting distribution histogram appears in Fig. 2. The number of stars in each $[\mathrm{O} / \mathrm{Na}]$ bin has been multiplied by $100 / 91$; i.e. an overall normalization to 100 stars has been applied, allowing one to read directly in the histogram percentages of the total. Thus, $30 \%$ of the stars in the rightmost bin have on average a composition of $[\mathrm{O} / \mathrm{Na}]=0.40$, i.e. the highest $\mathrm{O} / \mathrm{Na}$ ratio, which presumably reflects the original composition of the cluster. The remaining $70 \%$ of the stars have a composition modified to various degrees by the nucleosynthesis products of stars that evolved on relatively short timescales $\left(<10^{8} \mathrm{yr}\right)$, i.e. stars with initial mass $M>4 M_{\odot}$, according to the self-enrichment scenario (e.g. Ventura et al. 2001).

\section{Polluting agents and pollution scenarios}

Usually, it is claimed that hot-bottom burning (HBB) in massive asymptotic giant branch stars (AGBs) is responsible for the observed composition anomalies in GCs (Cottrell \& Da Costa 1981) ${ }^{4}$. Several custom-made detailed AGB models have recently been computed in order to test the HBB hypothesis (Ventura et al. 2001, 2002; Denissenkov \& Herwig 2003; Karakas \& Lattanzio 2003; Herwig 2004a,b; Ventura \& D'Antona 2005a,b,c). Most of these studies conclude that the AGB pollution scenario suffers from severe drawbacks from the point of view of the nucleosynthesis (e.g., Fenner et al. 2004; Charbonnel 2005). These difficulties stem from the subtle competition between HBB and third dredge-up, which contaminates the stellar envelope with the products of helium burning. The

\footnotetext{
${ }^{4}$ In their pioneer paper, Cottrell \& Da Costa (1981) suggest that $\mathrm{Na}$ and $\mathrm{Al}$ enrichment in $\mathrm{CN}$-strong stars of the GCs 47Tuc and NGC 6752 might be produced within intermediate-mass stars $\left(\sim 5-10 M_{\odot}\right)$. However, the invoked process is neutron-captures on ${ }^{22} \mathrm{Ne}$ and ${ }^{25} \mathrm{Mg}$ within the thermal pulse (the neutrons being released by the ${ }^{22} \mathrm{Ne}(\alpha, n){ }^{25} \mathrm{Mg}$ reaction; see Iben 1976).
} 


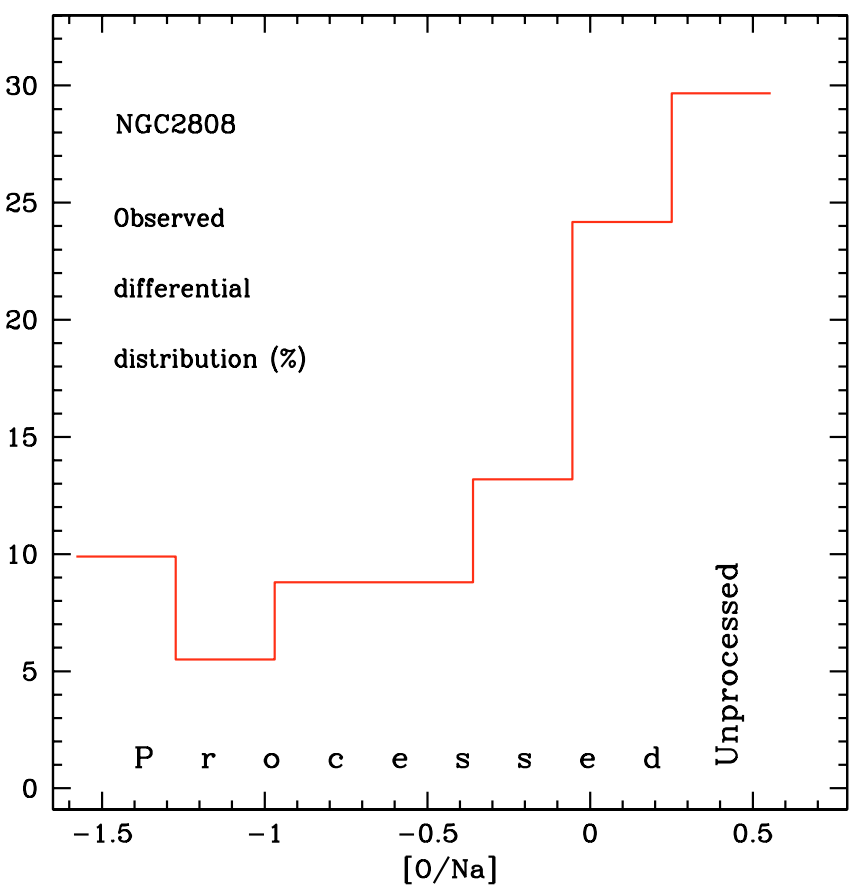

Fig. 2. Distribution histogram of stars of NGC 2808 (in percentages of total) as a function of $[\mathrm{O} / \mathrm{Na}]$; the distribution is calculated from data obtained by Caretta et al. (2006) (Sect. 2). Stars in the rightmost bin (30\%) are presumably born with the original (unprocessed) composition of the cluster $([\mathrm{O} / \mathrm{Na}]=0.40)$, while the remaining $70 \%$ have a composition processed to various degrees through $\mathrm{H}$-burning.

problems can be summarized as follows: (i) $\mathrm{O}$ is not depleted to the extent required by the observations, while $\mathrm{Na}$ is overproduced or over-destroyed depending on the treatment of convection; (ii) $\mathrm{Mg}$ is produced while it should be destroyed, and the $\mathrm{Mg}$ isotopic ratio is in conflict with the (rare) available data; (iii) $\mathrm{C}+\mathrm{N}+\mathrm{O}$ does not remain constant in AGB processed material, in contrast to observations (Dickens et al. 1991; Smith et al. 1996; Smith et al. 2005; Ivans et al. 1999). Rotationinduced mixing makes the situation even worse (Decressin \& Charbonnel 2005; Decressin et al., in preparation) by strongly enriching the envelope of intermediate-mass stars in O, already during the second dredge-up event. Last but not least, it is sometimes claimed that massive AGB stars are not expected to pollute the intracluster gas with s-process elements, in agreement with the observational requirements obtained by James et al. (2004) and presented in Sect. 1; indeed, lower-mass AGBs (1.5-3 $\left.M_{\odot}\right)$ are commonly considered to be the main source of s-elements in the Galaxy. However, s-process nucleosynthesis (from neutrons released by $\left.{ }^{22} \mathrm{Ne}(\alpha, n)\right)$ may certainly occur if temperatures in the thermal pulses of massive AGB stars are large enough (e.g. Goriely \& Siess 2005, and references therein). Very few quantitative studies have been devoted to that point, which clearly deserves additional investigation. Until the question is settled, it should not be taken for granted that massive AGBs do not produce s-elements at all; in our opinion, this constitutes another potential problem with their role as polluters of GCs.

Although it is too early to discard the AGB hypothesis, we feel that hydrogen burning in rotating massive stars may offer an interesting alternative (Decressin et al. 2006). In fact, as we argue in Sect. 4, this alternative may be more attractive, as far as the constraints on the IMF are concerned; other advantages of that idea are qualitatively discussed in Sect. 5. In the following, we explore both possibilities for the mass range of the polluting stars, namely, we consider AGB stars of masses 4-9 $M_{\odot}$ and massive stars of mass $>10 M_{\odot}$.

We explore two scenarios of the self-enrichment, which differ by the degree of nuclear processing in the ejecta of the polluters and thus by the nature of the gas (i.e., pure stellar ejecta or mixture of ejecta with gas of original composition) out of which the low-mass stars still surviving today were formed. In this section we present the underlying assumptions in detail and we evaluate the amount of ejecta vs. the amount of the long lived stars required in each scenario. Then in Sect. 4 we derive the corresponding constraints on the IMF for each scenario, in the cases where either intermediate or massive stars are the polluters.

\subsection{Scenario I}

The first scenario that we consider is the simpler one which assumes that:

I-a) stars with masses between 0.1 and $100 M_{\odot}$ are formed from gas of "normal" composition (i.e. the same composition as the one of field halo stars at that time, meaning for instance that $[\mathrm{O} / \mathrm{Na}]=0.4$ ) and with an IMF that has to be determined;

I-b) the ejecta of some (rapidly evolving) sub-class of those stars are polluted to various degrees by internal nucleosynthesis (i.e. the $[\mathrm{O} / \mathrm{Na}]$ ratio in the ejecta varies between -1.4 and 0.25 ), with an abundance distribution given by Fig. 2;

I-c) the ejecta are retained fully in the cluster and form directly a second generation of stars with mass $0.1-0.8 M_{\odot}$ (i.e., without being mixed with some original matter), the same IMF as the one of the first generation low-mass stars, and with a composition obviously different from the original one (as far as the H-burning products are concerned);

I-d) among the long-lived stars that we see today (with masses in the $0.1-0.8 M_{\odot}$ range) in NGC $2808,30 \%$ are formed from the original gas and $70 \%$ from the polluted ejecta.

We shall see that this "Scenario I" puts heavy constraints on the shape of the original IMF of the polluting stars, requiring it to be much flatter than the one observed today in Galactic and extragalactic stellar clusters. Indeed, it implies that the mass of the polluted ejecta should be about 2.3 times higher $(70 / 30=2.33)$ than the total mass of the first generation stars that survive today (mass between 0.1 and $0.8 M_{\odot}$ ). Moreover, if AGBs are assumed to be the polluting agents, we show that this scenario leads to the concomitant production of a large number of stellar remnants (mostly white dwarfs), the mass of which should be higher than the stellar mass of the cluster today. In contrast, if massive stars are assumed to be the polluting agents, the mass of stellar remnants is found to be considerably reduced. On the other hand, this scenario appears rather reasonable from the stellar nucleosynthesis point of view, since it invokes ejecta contaminated to various degrees (including very small ones) by the products of H-burning.

\subsection{Scenario II}

Our second scenario assumes that:

II-a) stars with masses between 0.1 and $100 M_{\odot}$ are formed from gas of "normal" composition (i.e. the same composition as the one of field halo stars at that time, meaning for instance that $[\mathrm{O} / \mathrm{Na}]=0.4)$ and with an $\mathrm{IMF}$ that has to be determined (identical to I-a); 
II-b) the ejecta of some (rapidly evolving) sub-class of those stars are polluted to an extreme degree by internal nucleosynthesis (i.e. the $[\mathrm{O} / \mathrm{Na}]$ ratio in the ejecta is invariably -1.4);

II-c) the timescale of the evolution of the polluters is shorter than the formation timescale of the low-mass stars (0.1-0.8 $M_{\odot}$ ), i.e. shorter than about 100 Myr (Stahler $\&$ Palla 2005). Then, the (extremely-processed) ejecta are mixed to various degrees with the gas of low-mass protostars of original composition, which are still forming;

II-d) among the long-lived stars that we see today (with masses in the $0.1-0.8 M_{\odot}$ range) in NGC $2808,30 \%$ are formed from the original gas (not contaminated at all by the ejecta) and $70 \%$ from various mixtures of the extremely-processed ejecta with the original gas.

Obviously, the starting and ending points of the two scenarios are identical (as they should be since both must account for the observed abundance distribution in Fig. 2). They differ in the intermediate steps. Scenario I involves two clearly distinct stellar generations, the second of which is exclusively made by the nuclearly processed ejecta of the first one. Scenario II involves only one stellar generation, the low-mass stars of which $\left(0.1-0.8 M_{\odot}\right)$ are contaminated at formation and, to various degrees, by the extremely-processed ejecta of their more massive and rapidly evolving sisters.

The assumption of extreme processing of the ejecta in Scenario II is neither mandatory (ejecta with various degrees of processing could also be assumed, as in Scenario I) nor easy to justify on physical grounds, as we discuss in Sect. 5. However, it is made here for two reasons:

a) combined with the abundance distribution diagram of NGC 2808 (top panel of Fig. 3), it allows one to evaluate the degree of mixing between processed and original material in the various $[\mathrm{O} / \mathrm{Na}]$ bins in a well-defined way (see middle and bottom panels of Fig. 3 and the next paragraphs);

b) precisely because the composition of the ejecta is so extreme, less material of processed composition is required for the mixture. Consequently, the constraints on the IMF (from the required ratio between mass of ejecta and mass in low-mass stars of $0.1-0.8 M_{\odot}$ ) are less severe than in Scenario I, as we show hereafter.

At this point, it should be stressed that our assumption that only stars of 0.1 to $0.8 M_{\odot}$ are formed from the ejecta (points I-c and II-c) is also made only in order to minimize the constraint on the IMF of the polluters (since their ejecta are used most efficiently, by forming exclusively stars still visible today). If stars with $M>0.8 M_{\odot}$ are assumed to also be formed from the ejecta, the required ejecta mass would still be larger, and the corresponding IMF of the polluters even flatter, than the ones we derive in the next section.

In order to evaluate the amount of ejecta with extremelyprocessed composition in the framework of Scenario II, we proceed as follows: assuming a mixture of unit mass of extremelyprocessed matter (mass fraction of a given element $X_{\text {EProc }}$ ) with $f$ parts of original matter (mass fraction $X_{\text {Orig }}$ ), the corresponding mass fraction $X_{\text {Mixt }}$ in the mixture will be:

$X_{\text {Mixt }}=\frac{X_{\text {EProc }}+f X_{\text {Orig }}}{1+f}$.

For the ratio of two elements $a$ and $b$ one then has

$\left(\frac{X_{a}}{X_{b}}\right)_{\text {Mixt }}=\frac{X_{a, \text { EProc }}+f X_{a, \text { Orig }}}{X_{b, \text { EProc }}+f X_{b, \text { Orig }}}$

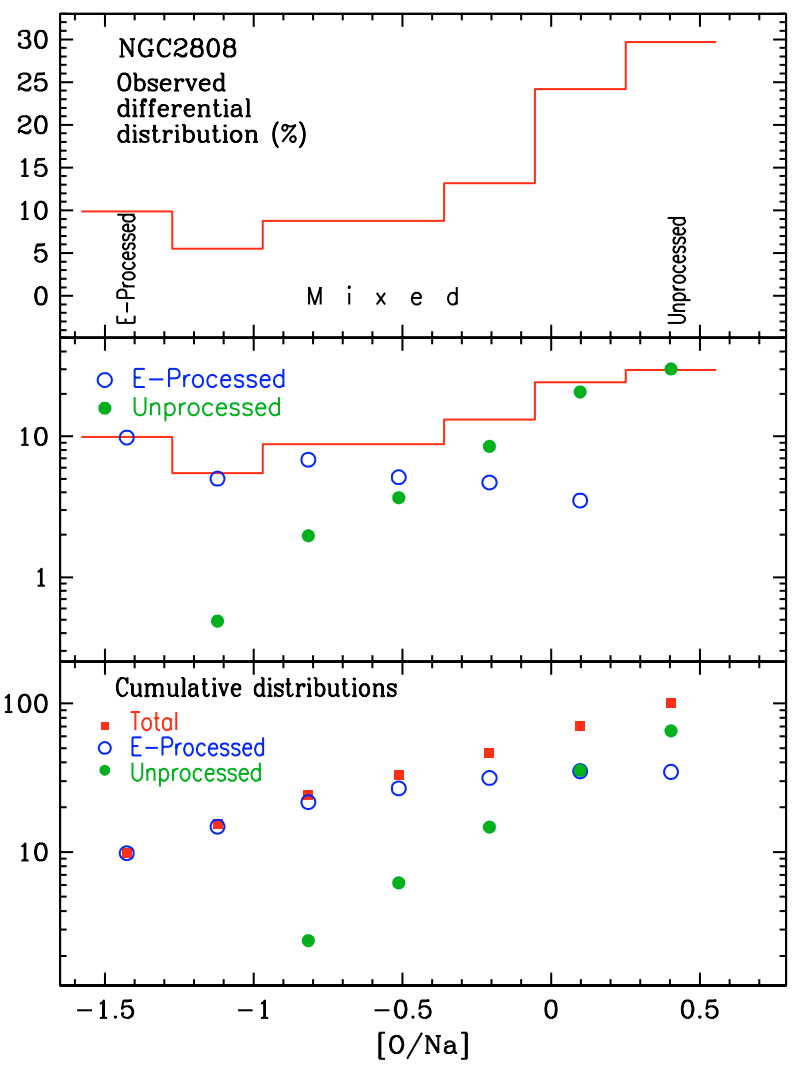

Fig. 3. Top: distribution histogram of stars of NGC 2808 (in percentages of total) as a function of [O/Na] (same as in Fig. 2). Stars in the rightmost bin (30\%) are presumably born with the original (unprocessed) composition of the cluster $([\mathrm{O} / \mathrm{Na}]=0.40)$, while the remaining $70 \%$ have a contaminated composition. Scenario II (see text) assumes that the mixed composition is obtained by mixing extremely-processed material (E-processed, with $[\mathrm{O} / \mathrm{Na}]=-1.40$, leftmost bin) with an unprocessed one, to various degrees, as explained in Sect. 3.2. Middle: open and filled circles indicate the amount of extremely-processed $\left(p_{i}\right)$ and unprocessed $\left(u_{i}\right)$ matter in each bin, respectively, while the histogram is the same as in the top panel (but on a logarithmic scale). Bottom: cumulative distributions of total (squares), extremely-processed (open circles), and unprocessed matter (filled circles), starting from the leftmost bin. Final values on the right are $P=35 \%$ for the extremely-processed and $U=65 \%$ for the unprocessed amounts of matter (see Sect. 3.2).

from which one may recover the dilution factor $f$ as

$f=\frac{X_{a, \text { EProc }}-\left(X_{a} / X_{b}\right)_{\mathrm{Mixt}} X_{b, \mathrm{EProc}}}{\left(X_{a} / X_{b}\right)_{\mathrm{Mixt}} X_{b, \text { Orig }}-X_{a, \text { Orig }}}$

as a function of the abundance ratio $\left(X_{a} / X_{b}\right)_{\text {Mixt }}$, provided the abundances of $a$ and $b$ in the original and the processed material are given.

In the framework of our Scenario II, we obtain the abundances of $\mathrm{O}$ and $\mathrm{Na}$ in the original and processed material by finding the corresponding values in the rightmost and leftmost bins, respectively, of the adopted [O/Na] distribution. These values appear in Fig. 1, where the averages $[\mathrm{O} / \mathrm{Fe}]$ and $[\mathrm{Na} / \mathrm{Fe}]$ in each of the adopted $[\mathrm{O} / \mathrm{Na}]$ bins appear and are:

$[\mathrm{O} / \mathrm{Fe}]_{\text {Orig }}=0.38$,

$[\mathrm{Na} / \mathrm{Fe}]_{\text {Orig }}=-0.03$,

$[\mathrm{O} / \mathrm{Fe}]_{\text {EProc }}=-0.72$,

$[\mathrm{Na} / \mathrm{Fe}]_{\mathrm{EProc}}=0.70$. 
The metallicity of NGC 2808 is $[\mathrm{Fe} / \mathrm{H}]=-1.1$ (Carretta et al. 2006), which allows one to find the absolute abundances of $\mathrm{O}$ and $\mathrm{Na}$ in the original and processed material. Turning all abundances into mass fractions, one then may solve Eq. (3) and find the dilution factor $f_{i}$ for each of the $[\mathrm{O} / \mathrm{Na}]$ bins $(i=1, \ldots, 7)$ in Fig. 3. Obviously, in the leftmost bin (material of extremelyprocessed composition exclusively) one has by definition $f_{1}=0$, while in the rightmost bin (material of original composition only) very high values are found for $f_{7}\left(f_{7} \rightarrow \infty\right)$.

As mentioned in Sect. 2, the number of stars in each bin $\left(n_{i}\right)$ also represents the percentage of material with (mixed) abundance ratio $[\mathrm{O} / \mathrm{Na}]$, since the total has been normalized to 100 . The fraction

$p_{i}=\frac{n_{i}}{1+f_{i}}$

then gives the amount of extremely-processed material (i.e. with $[\mathrm{O} / \mathrm{Na}]=-1.4)$ in each bin, while the remaining

$u_{i}=n_{i}\left(1-\frac{1}{1+f_{i}}\right)$

represents the amount of unprocessed (original) material. These amounts (also expressed as percentages of the total) appear in the middle panel of Fig. 3. Obviously, in the leftmost bin $\left(f_{1}=0\right)$ one has $p_{1}=n_{1}$, while in the rightmost bin $u_{7}=n_{7}$. By summing the amounts of the processed and unprocessed material over the seven bins, one then obtains the corresponding total amounts as

$P=\Sigma p_{i}, \quad U=\Sigma u_{i}$.

Those quantities appear in the rightmost bin of the bottom panel of Fig. 3. There is $P=35 \%$ of material with extremelyprocessed composition and $U=65 \%$ with original composition in the surviving stars of NGC 2808, in the framework of our Scenario II.

\subsection{Summary}

The two scenarios considered here are sketched in Figs. 4 and 5. The numbers appearing in those figures are percentages of the final total mass in low-mass long-lived stars $\left(0.1-0.8 M_{\odot}\right)$, as derived from our previous analysis (see also Fig. 3). There is a big quantitative difference between the two scenarios, concerning the ratio of the processed ejecta mass to the mass of stars with original composition in the low-mass range. In Scenario I, this ratio is $2.33(=70 / 30)$, whereas in Scenario II it is much lower, only $0.54(=35 / 65)$. Of course, this difference stems from the different assumptions on the composition of the ejecta: processed to various degrees in Scenario I vs. extremely-processed in Scenario II.

\section{Constraining the IMF of the polluting stars in NGC 2808}

In this section we explore the constraints imposed on the stellar IMF by the ratio mass(ejecta)/mass(low-mass stars) inferred from our previous analysis.

\subsection{The IMF in globular clusters}

Globular clusters provide, in principle, an ideal sample for accurately evaluating the stellar IMF in the mass range $M \leq 0.8 M_{\odot}$ and down to the H-burning limit. Indeed, they are composed of

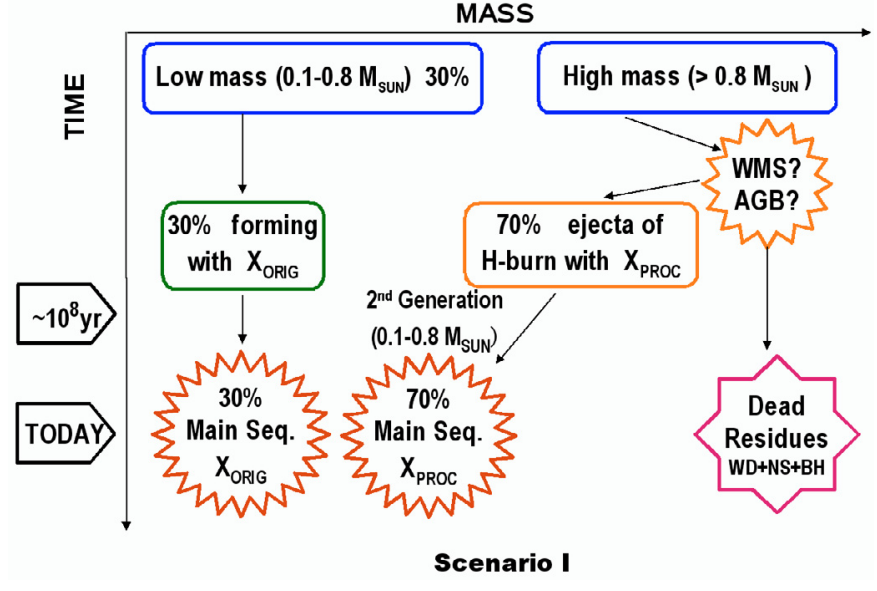

Fig. 4. Illustration of Scenario I with numbers corresponding to the analysis performed in Sect. 3.1. 30\% of the currently surviving lowmass stars of NGC 2808 are born with the original composition. The heavier stars of that first generation (either in the AGB stage, or through the winds of massive stars - WMS) eject 2.33 times more mass of varied composition, from which only low-mass stars (the remaining 70\%) is formed. This scenario puts relatively mild constraints on nucleosynthesis, but heavy ones on the initial mass function of the first stellar generation. Percentages refer to the total mass of low-mass stars of NGC 2808 (those still surviving today).

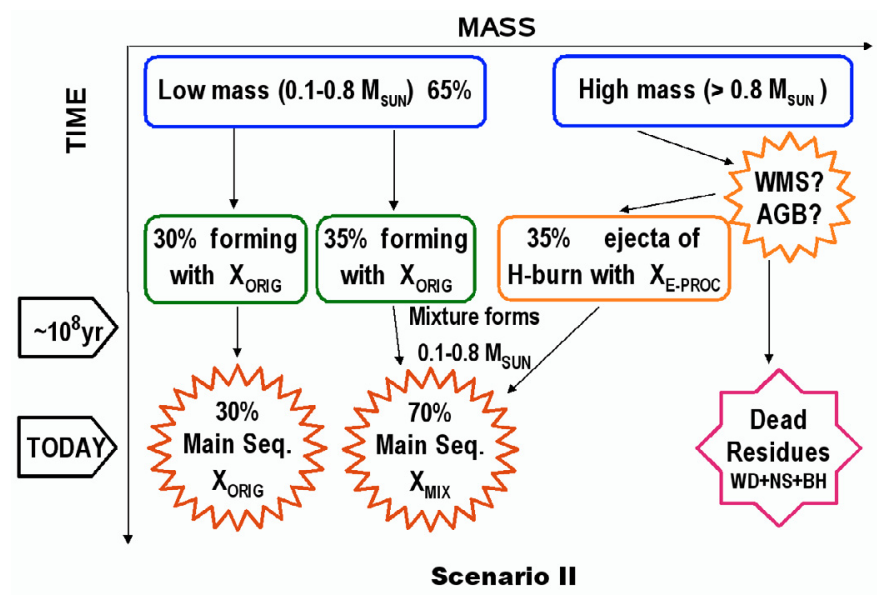

Fig. 5. Illustration of Scenario II, with numbers corresponding to the analysis performed in Sect. 3.2 and Fig. 3. 65\% of the material of currently surviving low-mass stars of NGC 2808 is of original composition $([\mathrm{O} / \mathrm{Na}]=0.40)$, and $35 \%$ is of extremely-processed composition $([\mathrm{O} / \mathrm{Na}]=-1.40)$, ejected from either AGB stars or massive stars. The latter material is mixed with $35 \%$ of the former to various degrees and forms low-mass stars (the currently surviving $70 \%$ stars of mixed composition), while the remaining $30 \%$ of the unprocessed material directly forms stars with the original composition. This scenario puts relatively mild constraints on the initial mass function, but puts extreme ones on the nucleosynthesis, since it requires all material ejected by the presumed sources (AGB or massive stars) to have $[\mathrm{O} / \mathrm{Na}]=-1.40$. Percentages refer to the total mass of the low-mass stars of NGC 2808 (those still surviving today).

stars with the same age, well-determined distances, and small variations in chemical composition (except for elements affected by H-burning) or in extinction within a cluster. Moreover, the fraction of binary stars outside the cluster core is less than $15 \%$ (e.g. Rubenstein \& Bailyn 1999) and affects the determination of the luminosity function very little. Once the luminosity function has been established, the existence of accurate stellar 
evolutionary models (reproducing well the observed colourmagnitude diagrams of clusters with metallicities $[\mathrm{Fe} / \mathrm{H}]<-1$, e.g. Pulone et al. 1998) allows one to derive the IMF of low-mass stars straightforwardly.

The major potential problem in this enterprise is that what we are formally measuring is the present-day mass function. This could differ from the IMF because of the dynamical evolution of the cluster, which may have both internal and external origins: internal, due to relaxation of the $N$-body system towards energy equipartition, by expelling less massive bodies towards the periphery of the cluster and accumulating massive ones in the centre; and external, due to tidal interactions with the Galactic potential (or molecular clouds, other clusters etc.), which may result in the loss of the less massive bodies. Both effects lead to the mass segregation of stars in space and time. Paresce \& de Marchi (2000, hereafter PdM00) find that the effects of mass segregation alter the stellar mass function both in the innermost regions (making it flatter than the IMF) and in those outside the half-light radius (making it steeper than the IMF); however, around the half-light radius, the effects of mass segregation are insignificant (see Sect. 4 and Fig. 4 in PdM00).

By using HST measurements of the luminosity functions at (or just beyond) the half-light radius of a dozen globular clusters, PdM00 find that the corresponding IMFs have the shape of a lognormal distribution characterized by a peak mass $M_{\mathrm{C}}=$ $0.33 \pm 0.03 M_{\odot}$ and a standard deviation $\sigma=0.34 \pm 0.04^{5}$. Their measurements cover the mass range $0.09-0.7 M_{\odot}$, but we assume here that the derived IMF shape is valid up to the turn-off mass of $\sim 12$ Gyr globular clusters, i.e. up to $\sim 0.8 M_{\odot}$. We then assume that in the mass range of low-mass stars $\left(0.1-0.8 M_{\odot}\right)$ the IMF has the form

$\Phi(M)=\frac{\mathrm{d} N}{\mathrm{~d} M} \propto \frac{1}{2 M}\left(\frac{\log \left(M / M_{\mathrm{C}}\right)}{\sigma}\right)^{2}$

with the values of $M_{\mathrm{C}}$ and $\sigma$ given in the previous paragraph. The IMF of more massive stars (living less than 12 Gyr) is not observable in globular clusters, but it is measured in young clusters, where it has been found that it can be approximated well by a power-law of the form

$\Phi(M) \propto M^{-(1+X)}$

with $X=1.35$ being the "classical" Salpeter (1955) value. As discussed in Kroupa (2002, and references therein), the Salpeter value fits the available data extremely well over a wide range of cluster masses and in different galaxies and with a dispersion of about 0.35 , i.e. $X=1.35 \pm 0.35$.

In this work we assume that the IMF of the globular clusters was a composite one, given by Eqs. (7) and (8) for the mass ranges $0.1-0.8 M_{\odot}$ and $0.8-100 M_{\odot}$, respectively. We allow the value of $X$ to vary in the range -0.25 to 1.35 and constrain it using the results of the analysis in Sect. 3. All IMFs are normalized to

$\int_{0.1}^{100} M \Phi(M) \mathrm{d} M=1$.

Two of the adopted IMFs, with $X=0.15$ and 1.35 , are shown in Fig. 6 (denoted with letters A, and B respectively).

5 This agrees well with various studies (Zinnecker 1984; Adams \& Fatuzzo 1996; Elmegreen 1999) that show that one would expect a lognormal form for the IMF if the number of independent parameters governing the fragmentation of a proto-stellar cloud is large enough $(>5)$.

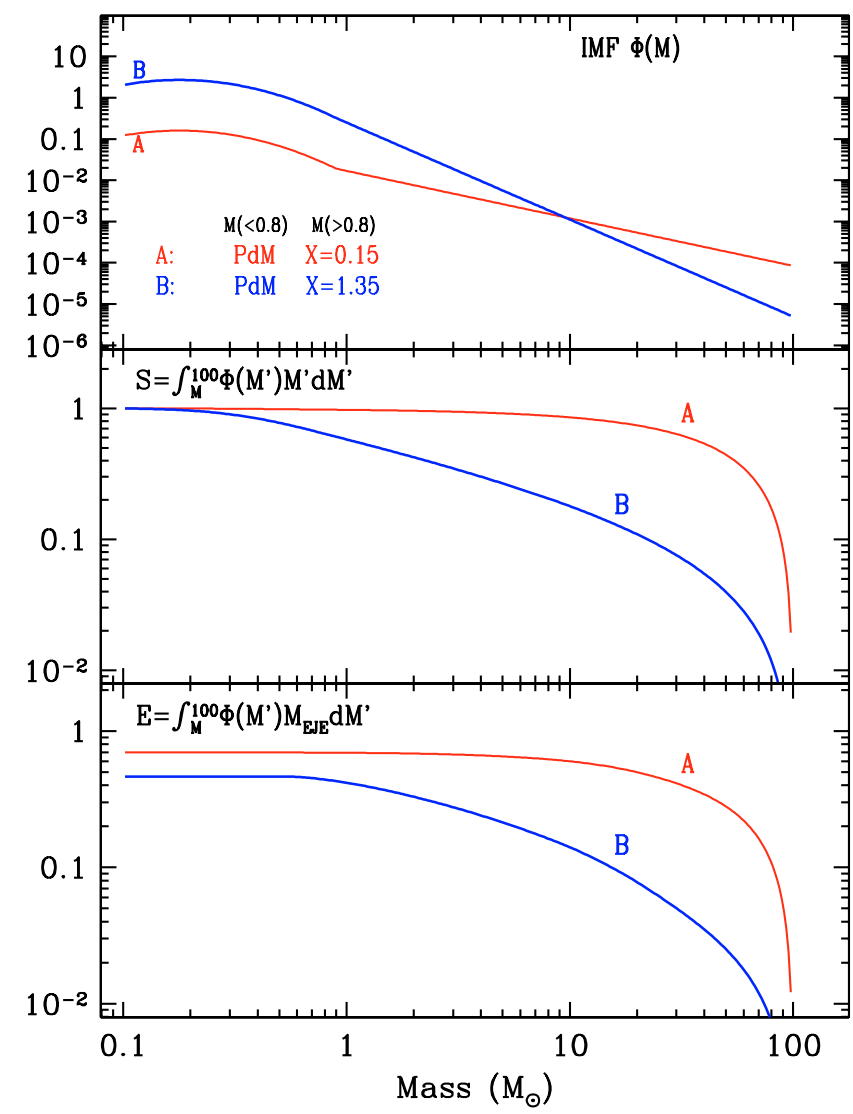

Fig. 6. Top: examples of the stellar IMFs studied in this work. They are composed of (i) the Paresce \& de Marchi (2000, PdM) IMF of Eq. (7) in the range $0.1-0.8 M_{\odot}$, and (ii) of the power-law IMF of Eq. (8) at $M>0.8 M_{\odot}$, with slope $X=0.15$ and 1.35 for IMF $A$ and $B$, respectively. All IMFs are normalized to $\int_{0.1}^{100} M \Phi(M) \mathrm{d} M=1$. Middle: corresponding total masses of the IMFs as a function of the lower integration limit $M$ in the integral $\int_{M}^{100} M^{\prime} \Phi\left(M^{\prime}\right) \mathrm{d} M^{\prime}$. IMF $A$ has more mass in heavy stars (and, correspondingly, less in low-mass stars) than IMF $B$. Bottom: corresponding ejecta mass fractions as a function of the lower integration limit $M$ in the integral $\int_{M}^{100} \varepsilon\left(M^{\prime}\right) \Phi\left(M^{\prime}\right) \mathrm{d} M^{\prime}$, where $\varepsilon(M)$ is the mass of the H-processed ejecta. Again, IMF $A$ has a much higher ejecta mass fraction in massive stars than IMF $B$.

\subsection{Mass of long lived stars, ejecta, and residues}

A couple of other interesting quantities appear in Fig. 6. The mass fraction in the form of stars heavier than a mass $M$ is

$S(M)=\int_{M}^{100} M^{\prime} \Phi\left(M^{\prime}\right) \mathrm{d} M^{\prime}$

where, by definition, $S(0.1)=1$. Flat IMFs have a higher fraction of their mass in heavy stars and a correspondingly lower fraction in low-mass stars than IMFs with a steep slope (see middle panel in Fig. 6). We also present (bottom panel in Fig. 6) the hereby defined ejecta mass fraction

$E(M)=\int_{M}^{100} \varepsilon\left(M^{\prime}\right) \Phi\left(M^{\prime}\right) \mathrm{d} M^{\prime}$,

where $\varepsilon(M)$ is defined as the part (in $M_{\odot}$ ) of the star that is processed only through $H$-burning and is returned to the ISM. Even with the help of detailed stellar models it is difficult to have precise values for $\varepsilon(M)$, since those values depend on several at 
present poorly known ingredients, such as mixing criteria, massloss rates, etc. In this work, we choose to deliberately maximize the amount of $\mathrm{H}$-processed ejecta from each star, in order to obtain upper limits to the slope of the corresponding IMF. For that purpose, we adopt the following $\varepsilon(M)$ relations:

$$
\begin{aligned}
& \varepsilon(M)=M-M_{\mathrm{Res}}(M) \quad\left(M<9 M_{\odot}\right) \\
& \varepsilon(M)=M-M_{\mathrm{He}}(M) \quad\left(M>9 M_{\odot}\right)
\end{aligned}
$$

where $M_{\text {Res }}$ is the mass of the residue (white dwarf for $M<$ $9 M_{\odot}$, neutron star or black hole otherwise) and $M_{\mathrm{He}}$ is the mass of the H-exhausted core of the star. This choice obviously maximizes the mass of the H-processed ejecta because it assumes that:

- in intermediate mass stars, all the mass outside the white dwarf remnant is processed exclusively through $\mathrm{H}$-burning: in reality, part of that mass is processed in the He-burning shell, but we assume here that this is negligible;

- in massive stars, all the mass outside the He-core is processed through H-burning; in reality, part of that mass is not processed at all (i.e. it is ejected with its original composition by the early stellar wind), while another part (close to the Hecore) is contaminated by He-burning products. Again, we assume those parts to be negligible, in order to maximize the mass of the H-processed ejecta.

In Fig. 7 we display the adopted $\varepsilon(M)$ (from Eqs. (12) and (13)), as well as $M_{\text {Res }}$, which is given from the relations:

$$
M_{\operatorname{Res}}(M)= \begin{cases}0.446+0.106 M & \left(M<9 M_{\odot}\right) \\ 1.5 M_{\odot} & \left(10<M / M_{\odot}<25\right) \\ 3 M_{\odot} & \left(M>25 M_{\odot}\right) .\end{cases}
$$

The white dwarf vs. initial mass relation for $M<9 M_{\odot}$ is from Iben \& Tutukov (1984), which assumes that stars in the 10-25 $M_{\odot}$ range produce neutron stars of $1.5 M_{\odot}$, while more massive ones produce black holes of $3 M_{\odot}$. The mass of the neutron star or the black hole affects the results discussed hereafter little, as far as it remains below the mass of the Hecore, which is a reasonable assumption.

The ejecta mass fraction $E(M)$ for the two IMFs $A$ and $B$ appear in the bottom panel of Fig. 6. Flat IMFs have higher ejecta mass fractions than IMFs with a steeper slope. Finally, in a similar way, the mass fraction locked in residues is calculated as

$R(M)=\int_{M}^{100} M_{\operatorname{Res}}\left(M^{\prime}\right) \Phi\left(M^{\prime}\right) \mathrm{d} M^{\prime}$.

From the defining Eqs. (9) to (15), one may calculate any quantity of interest for a given IMF; e.g. the mass locked in low-mass stars (in the range $0.1-0.8 M_{\odot}$ ) still surviving today is

$S(0.1-0.8)=S(0.1)-S(0.8)$,

while the ejecta mass fraction of stars in the 4 to $9 M_{\odot}$ range is

$E(4-9)=E(4)-E(9)$,

and similar relations hold for e.g. the mass of residues.

Four quantities of interest, calculated along the previous lines for all the IMFs studied here, are plotted in Fig. 8 (top

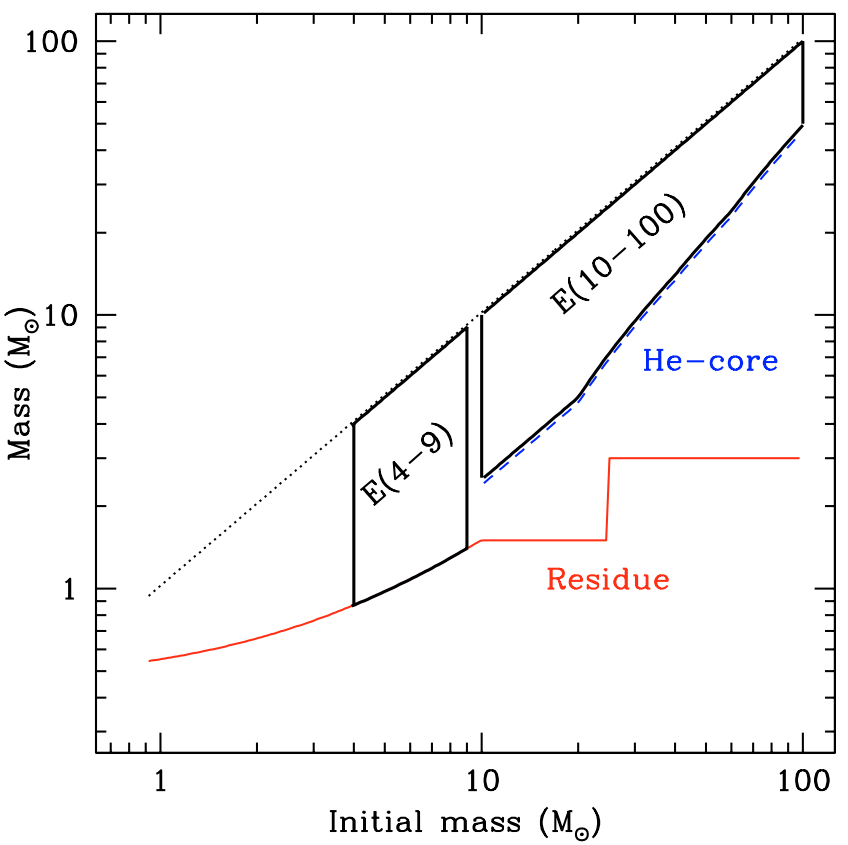

Fig. 7. Mass of He-core (dashed curve) for stars with $M>9 M_{\odot}$ and mass of stellar residues (solid curve) as a function of initial stellar mass. The dotted line indicates the initial stellar mass, while the areas within thick curves represent the $\mathrm{H}$-processed ejecta that may contribute to the self-enrichment of globular clusters; the indicated amount is clearly an overestimation (see text).

panel) as a function of the slope $X$ of the $M>0.8 M_{\odot}$ part of the IMF, which we wish to constrain. They are, respectively:

- the ejecta mass fraction $E$ from massive stars (10 to $100 M_{\odot}$ ). It is assumed that the envelopes of those stars are processed through $\mathrm{H}$-burning at high enough temperatures and are ejected by gently blowing stellar winds in the ISM, where they participate in the formation of new stars;

- the ejecta mass fraction $E$ from HBB in massive AGB stars (of 4-9 $M_{\odot}$ ); these ejecta escape their stars at relatively low velocities and are trapped in the cluster and form new stars. It is assumed here that the total envelope mass outside the carbon-oxygen white dwarf is processed that way;

- the mass fraction $R$ in stellar residues (mostly white dwarfs) from all stars present in the original IMF that evolved until today (i.e. stars in the 0.8 to $100 M_{\odot}$ range);

- the mass fraction $S$ in long-lived stars $\left(0.1-0.8 M_{\odot}\right)$.

The first of these quantities increases when the slope of the highmass part of the IMF decreases (when the IMF becomes flatter), while the opposite happens with the last quantity; the other two are relatively insensitive to the IMF slope.

\subsection{Constraints on the IMF of NGC 2808}

Armed with these numbers, one may now explore the implications for NGC 2808 in the framework of the self-enrichment scenario. In the middle panel of Fig. 8 the ratio of the H-processed ejecta mass to the mass locked in low-mass stars of the original IMF (stars that still survive today) is plotted vs the slope $X$. Two cases are considered, namely, that the ejecta originate either in massive stars (with mass $10-100 M_{\odot}$ ) or in intermediate mass stars (AGBs of 4-9 $M_{\odot}$ ). Except for the steepest IMFs, the former ratio is always higher than the latter (since the ejecta mass 
of $10-100 M_{\odot}$ is higher than the ejecta mass of $\left.4-9 M_{\odot}\right)$. Of course, restraining the mass range of massive stars that could contribute to the H-processed ejecta (assuming e.g. that only stars in the 30-100 $M_{\odot}$ range develop sufficiently high temperatures for the operation of $\mathrm{Ne}-\mathrm{Na}$ or $\mathrm{Mg}-\mathrm{Al}$ cycles) or assuming a lower mass for the processed envelopes of massive stars (instead of the Eq. (13) adopted here) would reduce the ejecta mass fraction of massive stars accordingly and would bring down the corresponding curves in Fig. 8 (top and middle panels). The same, of course, holds for the contribution of AGBs, which could well be lower than assumed here (for instance, if the mass of their $\mathrm{H}$-processed ejecta is less than assumed in Eq. (12)). Thus, the results presented in the middle panel of Fig. 8 should clearly be considered only as upper limits to what AGBs or massive stars might do. In reality, all curves involving ejecta $E$ in the top and middle panels of Fig. 8 should be shifted downwards.

Those theoretical results are compared to the observationally inferred requirements for NGC 2808 for both Scenarios I and II in Fig. 8 (middle panel). As found in Sect. 3, Scenario I requires a ratio of $\mathrm{H}$-processed ejecta to mass in low-mass stars with an original composition of $70 / 30=2.33$, while Scenario II requires a lower ratio, of 35/65 $=0.54$. As can be seen from Fig. 8 (middle panel), the requirement of Scenario I is only satisfied for slopes $X<0.8$ in the case of massive stars and $X<0.15$ for the massive AGBs. The requirement of Scenario II is satisfied for $X<1.25$ in the former case and for $X<0.95$ in the latter. Those IMFs with the standard slope $X=1.35$ fail to satisfy observational requirements by a factor of 2 in our Scenario II and by a factor of 10 in our Scenario I.

A diagram such as the one in the middle panel of Fig. 8 should offer a way to determine the slope of the high-mass $\left(>0.8 M_{\odot}\right.$ ) IMF of a globular cluster with a measured abundance distribution such as NGC 2808. However, this determination is made in the framework of models with extreme assumptions about both the polluter ejecta and their mixing with the pristine gas. In Sect. 5 we discuss these assumptions and conclude that the IMF should be much flatter (i.e. $X$ should be much smaller) in a realistic scenario than determined in the middle panel of Fig. 8. Still, it will be model-dependent since the necessary ingredients (amount and composition of ejecta and degree of their mixing with the ISM) cannot be constrained independently at present.

At this point, it should be noted that the IMF may critically affect the fate of a dense stellar cluster. Simulations of the dynamical evolution of GCs, including the effects of tidal stripping and of mass loss from stellar evolution, show indeed that a flat mass spectrum speeds up the cluster disruption (e.g., Joshi et al. 2001; Giersz 2001). This potential problem is alleviated in the present self-enrichment scenario, because the mass lost by the polluters is assumed to be incorporated in the low-mass stars of the cluster. Nevertheless, the self-enrichment scenario needs to be tested quantitatively with realistic dynamical evolution models.

\subsection{Consequences for the amount of stellar residues}

One way to further constrain the various scenarios is by searching for signatures left over in the form of stellar residues. In the bottom panel of Fig. 8 we explore the consequences of our different assumptions for the mass of "dark objects" in NGC 2808, namely residues of stars with initial mass $M>0.8 M_{\odot}$. Their mass (determined by the assumed IMF from Eq. (14) and given in Fig. 8, top panel) is compared to the total mass of the longlived stars $\left(M \leq 0.8 M_{\odot}\right)$ of the cluster. The total mass is the sum of the original (unpolluted) low-mass stars $S(0.1-0.8)$ plus

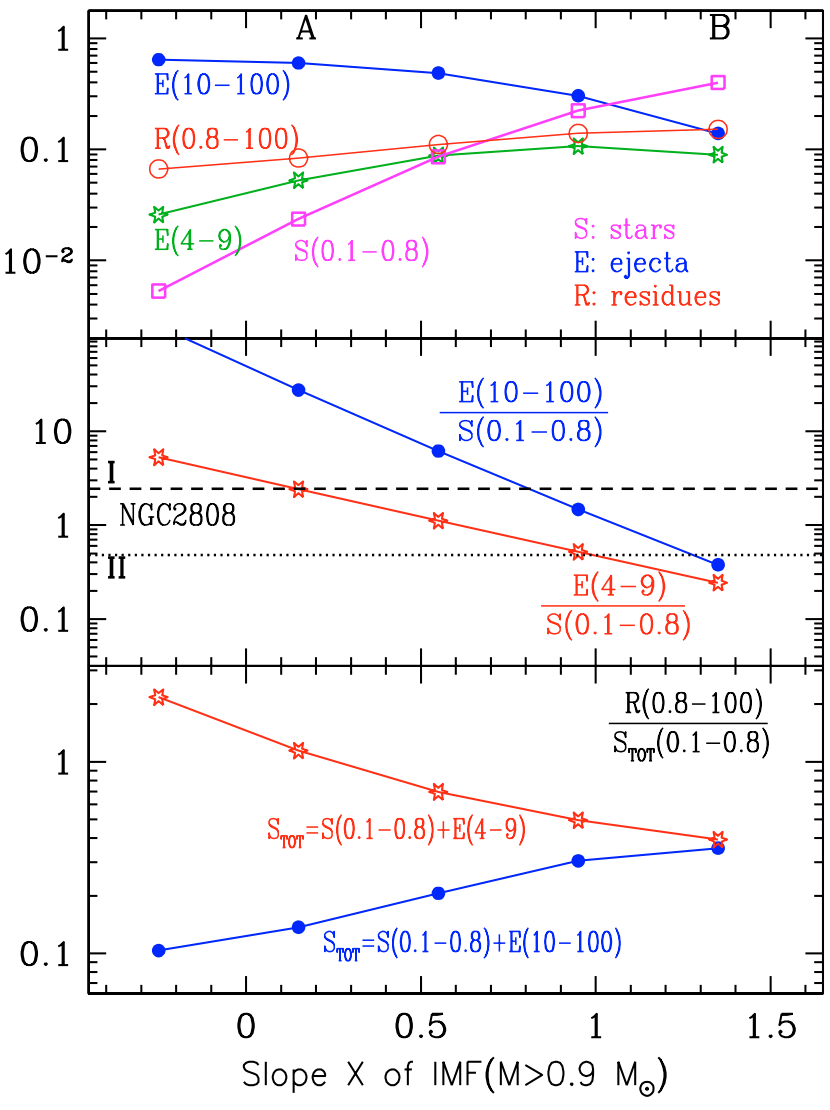

Fig. 8. Top: mass fraction in low-mass stars surviviving today ( $S\left(0.1-0.8 M_{\odot}\right)$, open squares $)$, mass fraction $R\left(0.8-100 M_{\odot}\right)$ in stellar residues (i.e. white dwarfs, neutron stars, or black holes from stars of initial mass $M>0.8 M_{\odot}$, open circles), and H-processed ejecta mass fractions $E$ from stars in the 4-9 $M_{\odot}$ (asterisks) and 10-100 $M_{\odot}$ ranges (filled circles), as a function of the slope $X$ of the high-mass part of the IMF. For the low-mass part, $M \leq 0.8 M_{\odot}$, the PdM00 IMF of Eq. (7) is always assumed. Letters A and B denote the results for the IMFs used in Fig. 6. Middle: ratio of ejecta mass (of either 4-9 $M_{\odot}$ or $10-100 M_{\odot}$ stars) to the mass of low-mass stars $S(0.1-0.8)$ for each of the IMFs of our study. Note that $S$ represents stars (or material) of only original (unprocessed) composition. The two horizontal lines correspond to the constraints imposed by the analysis of Sect. 2.2 and Figs. 2 and 3, according to Scenarios I (dashed) and II (dotted), respectively. Bottom: ratio of residue mass to the total mass of low-mass stars surviving today, for each of the IMFs studied. The total mass is the sum of the stars formed from original (unprocessed) material plus those formed from the ejecta of either AGBs (4-9 $M_{\odot}$ ) or massive stars $\left(10-100 M_{\odot}\right)$.

the polluted ones. If the polluted long-lived stars originate in the ejecta of 10-100 $M_{\odot}$ stars (mass $E(10-100)$ ), the sum increases considerably at low $X$ values, and the ratio of residues to stars decreases in consequence. In contrast, if the polluted long-lived stars originate in the ejecta of 4-9 $M_{\odot}$ stars (mass $E(4-9)$ ), the sum decreases at low $X$ and the mass of the residues becomes comparable to the mass of active stars today, especially in case of Scenario I. Thus, the mass ratio of residues to long-lived stars is sensitive to the assumption made about the mass range of the polluters, especially for flat IMFs (such as those required to explain the abundance distribution of NGC 2808, and presumably of other clusters as well).

Unfortunately, such a ratio has not been determined yet for the case of NGC 2808. However, in the case of the cluster M4 (another mildly metal-poor GC with $[\mathrm{Fe} / \mathrm{H}] \sim-1.08$; Ivans et al. 2001), the number ratio of white dwarfs to long-lived stars has 
been determined by Richer et al. (2002, hereafter RBF02). They used HST observations of that cluster to probe its luminosity function (LF) down to the H-burning limit $\left(\sim 0.09 M_{\odot}\right)$. Using stellar models of low-mass and low-metallicity stars (which still suffer from important uncertainties), they transformed the LF into an MF and concluded that the latter can be fitted by a powerlaw of slope $\alpha=0.75$ (or $X=\alpha-1=-0.25$ in the notation adopted here) in the mass range $0.09-0.65 M_{\odot}$. In a subsequent work, however, de Marchi et al. (2004) argue that the use of more appropriate stellar models leads to a mass function similar to the one that PdM00 had determined for 12 GCs (Eq. (7)). In the following we also adopt this low-mass MF (the same used throughout our paper) for M 4 .

By applying substantial completeness corrections to their sample, RBF02 derive the number of main sequence stars in the range 0.09 to $0.65 M_{\odot}$ and the number of white dwarfs from progenitor stars in the range 0.8 to $8 M_{\odot}$ : they are, respectively, $N_{\mathrm{MS}}(0.09-0.65)=570$ and $N_{\mathrm{WD}}=602$. The number ratio of white dwarfs to long lived stars is thus $\sim 1$. It should be stressed that these numbers are determined essentially by the applied completeness corrections, rather than by the observations (see Fig. 3, top panel, in RBF02).

RBF02 find that a single-slope power-law IMF, with $\alpha=$ 1.05 (or $X=0.05$ ), i.e. the steepest slope compatible with their data, can accommodate this high ratio of $N_{\mathrm{WD}} / N_{\mathrm{MS}}$. They assume, of course, that both the observed MS stars and the WDs come from the original (first generation) IMF of the cluster; i.e., they do not consider long-lived stars formed from the ejecta of the first generation. Since M4 is a cluster with observed O vs. $\mathrm{Na}$ anticorrelation (Ivans et al. 1999, 2001), their IMF determination has meaning only under the assumption that the observed anticorrelation is due to internal evolution and not to self-enrichment.

For a composite IMF, kethe one adopted here (with PdM00 IMF below the turn-off mass of $0.8 M_{\odot}$ and a power law above it), we find that a slope $X=-0.3$ above the turn-off mass is required in the internal evolution scenario to reproduce the derived $N_{\mathrm{WD}} / N_{\mathrm{MS}} \sim 1$ (middle panel of Fig. 9).

Somewhat surprisingly, in the self-enrichment scenario (middle and bottom panels of Fig. 9), such a high $N_{\mathrm{WD}} / N_{\mathrm{MS}}$ ratio is never obtained: values around 0.2 are obtained if the polluters are assumed to be AGBs and much lower ones if the polluters are massive stars. This may appear paradoxical, since at small IMF slopes and in the case of AGBs as polluters, residues dominate long-lived stars by mass, as clearly seen in Fig. 9 (bottom panel). This apparent discrepancy is due to the fact that the ejecta mass of a e.g. $4 M_{\odot}$ star can give rise to a dozen or so low-mass stars $\left(\sim 0.3 M_{\odot}\right)$ of 2 nd generation; thus, the resulting number ratio between WDs and long-lived stars is much lower than the corresponding mass ratio between WDs and ejecta. Interestingly, in the case of internal evolution (implicitly adopted in RBF02), the residues should completely dominate the long lived stars by mass in M 4, by a factor of 10 , as can be seen in the bottom panel of Fig. 9 due to the fact that each residue (white dwarf, neutron star, or black hole) is several times more massive than a typical low-mass star.

The low number ratio of white dwarfs over low-mass stars obtained in our self-enrichment scenarios (lower than derived in M4), does not necessarily point to a fatal flaw for those scenarios. It may well be that the ejecta mass (and the resulting number of second generation stars) is lower than assumed here, in which case a ratio closer to the observed one will be obtained. Such a reduced ejecta mass is, in fact, quite plausible (see discussion in Sect. 5.1).

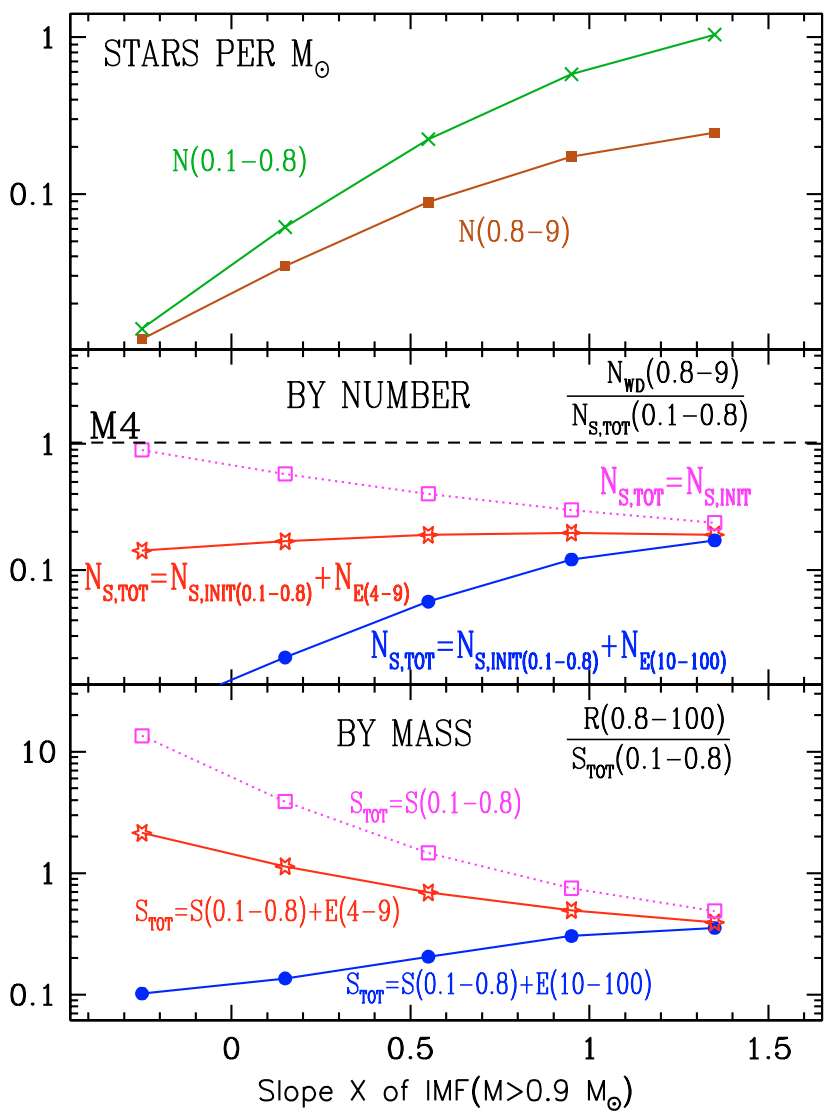

Fig. 9. Top: number of stars per unit solar mass, as a function of the slope $X$ above the turn-off mass. The two curves corerspond to longlived stars $\left(0.1-0.8 M_{\odot}\right)$ and to stars leaving behind white dwarfs (0.8-9 $M_{\odot}$ initially). Middle: number ratio of white dwarfs to longlived stars for the various IMFs studied in this work. The two solid curves correspond to the cases where the polluters are AGBs (asterisks) and WMS (filled circles), as in the middle and bottom panels of Fig. 8. The dotted curve is obtained by simply dividing the two curves of the top panel, i.e. no creation of $0.1-0.8 M_{\odot}$ stars by the ejecta of polluters (self-enrichment) is considered. The horizontal dashed line represents the results of the study of Richer et al. (2002) for M 4. Bottom: curves as in the middle panel, but for mass ratios instead of number ratios. Also, the residues include not only white dwarfs (as in the middle panel) but also neutron stars and black holes.

\subsection{Constraints from the horizontal branch in NGC 2808}

A determination of the polluter IMF on the basis of star counts has been also attempted recently by D'Antona \& Caloi (2004), who noted that AGB winds are helium-enriched (mainly as a consequence of the second dredge-up; Ventura et al. 2001). The second generation stars formed from AGB ejecta should then have a higher helium content than the first generation stars, which were born with the primordial cluster helium abundance. This difference in helium may substantially alter their advanced evolution.

D'Antona et al. (2002) evaluates the impact of a spread in helium within the low-mass star population on a GC colour-magnitude diagram and discusses the evolutionary properties of GC stars in which variations of the initial helium are allowed. They show that within reasonable limits ( $Y$ varying between 0.24 for the proto-cluster abundance and 0.28 for the most polluted stars), the impact on the main sequence, turnoff, and RGB would be low and undetectable observationally. Also, the 
horizontal branch luminosity appears to be only slightly affected. As a consequence the age determination should only be modified in a limited way. However, the differences in the morphology of the horizontal branch would be noticeable; in particular, the He enrichment could play a role in the formation of blue tails.

Following this idea, D'Antona \& Caloi (2004, hereafter DC04) studied the morphology of the horizontal branch of NGC 2808 in detail. It is composed of two well-separated regions: a red clump and an extended blue tail starting from the blue side of the RR Lyrae gap and reaching below the cluster turn-off (Bedin et al. 2000, and references therein). DC04 shows that the dichotomy of the horizontal branch of NGC 2808 can be reproduced by assuming different helium contents for the clump and blue HB stars and argues that this unveils the signature of two separate star formation events in the early cluster evolution. Within their self-enrichment framework (similar, but not identical, to our Scenario I), the red clump stars belong to the first stellar generation formed with the original helium, while secondgeneration stars born directly from the ejecta of AGB stars of various masses (and thus with various He-enrichments) populate the blue part of the HB. The lack of stars in the RR Lyrae region is attributed to the gap in helium between the two stellar generations ${ }^{6}$.

DC04 further explore the implications of their hypothesis by deriving the IMF of the first stellar generation based on star counts along the HB of NGC 2808. In order to explain the number of HB blue stars, they adopt a relation between the progenitor AGB mass and the helium content of their ejecta (based on stellar models) and derive a relatively flat IMF, with $X \sim 0$. This value is very close to the upper limit we derived in Sect. 4.3 for AGB stars being the polluters within Scenario I.

Helium enrichment is a natural outcome of AGB models. However, the results of DC04 are extremely model-dependent; they rely in particular on a simple linear relation between the average helium content in the ejecta and the progenitor mass, require a fine-tuning of the mass loss on the RGB, and depend on delicate transformations between the theoretical and observational plane. Additionally, other phenomena may intervene in the building of the colour-magnitude diagram morphology of a GC (mass-loss processes, stellar multiplicity, stellar rotation, dynamical effects), which should be ultimately taken into account in such an analysis (see e.g., Sosin et al. 1997; Castellani et al. 2006, and references therein).

Claims of supporting evidence for the presence of heliumrich populations in GCs were recently obtained through the detailed analysis of various colour-magnitude diagram features (e.g., Lee et al. 2005, and references therein). This type of analysis newly supports the self-enrichment hypothesis, but sheds no light on the nature of the polluters, since both AGBs and WMS contribute a lot of $\mathrm{He}$, as discussed in Sect. 5.

The results presented and discussed in this section illustrate how deep-counting observations of GC stars may be used to constrain the massive star (=polluter) IMF of the GCs in the framework of the self-enrichment scenario. It is true that the

${ }^{6}$ D'Antona et al. (2005) push the analysis of the CMD of NGC 2808 further. They propose that the main sequence width of this $\mathrm{GC}$ can be explained by the presence of three stellar generations born with different helium contents: $Y \sim 0.24$ (first generation born with proto-cluster abundance), $Y \sim 0.4$ (second generation, in chronological order, born from the winds of the most massive AGBs of the first generation), and $Y \sim 0.26-0.29$ (third generation born from the ejecta from less massive AGBs of the first generation). In their scenario, late binary type II supernova explosions are invoked to explain why there are no stars formed with $Y$ between 0.29 and 0.4 . simplifying assumptions made in our work (upper mass limits for the IMFs of the first $-100 M_{\odot}-$ and second $-0.8 M_{\odot}-$ stellar generations; same form for the low mass part of the IMFs in both stellar generations) are not unique, and a degenerate set of constraints would be obtained in a detailed analysis. However, with larger observational data sets available for a given cluster (including e.g. abundance distributions for several elements, number counts of white dwarfs and low mass stars - including those of the horizontal branch, dynamical determination of the baryonic mass of the cluster etc.) and with improved theoretical understanding of stellar nucleosynthesis issues (e.g. amount and composition of ejecta) this degeneracy will ultimately be lifted.

\section{Discussion}

Today, there is neither a unique, a well-defined, nor even a fully self-consistent scenario for the self-enrichment of globular clusters. The most commonly-invoked one assumes that the $\mathrm{H}$-processed ejecta of massive AGB stars of a first stellar generation (of unspecified, but presumably flatter-than-normal, IMF) form a second stellar generation (of equally unspecified IMF) and of appropriate chemical composition (i.e. in agreement with observed $\mathrm{O} / \mathrm{Fe}$ vs. $\mathrm{Na} / \mathrm{Fe}$ ratios in $\mathrm{GC}$ stars). Most of the theoretical effort up to now has been devoted to trying to reproduce the observed $\mathrm{O}$ vs. $\mathrm{Na}$ or $\mathrm{Mg}$ vs. Al relations from the nucleosynthesis point of view (see references in Charbonnel 2005).

In this section, we discuss several aspects of the two selfenrichment Scenarios (I and II), which are sketched in Sect. 3 and which involve not only AGBs but also winds of massive stars as alternative sources of the chemically polluted material.

\subsection{The mass and composition of the polluting ejecta}

We start by noting that any scenario of that kind necessarily involves assumptions about three quantities:

a) the IMF (mass limits and shape) of the first stellar generation and, when it exists, of the second one (there are two generations in our Scenario I but only one in our Scenario II);

b) the amount and composition of the stellar ejecta that contribute to the formation of the contaminated stars, as well as the mass range of the polluting stars;

c) the amount of unprocessed gas, which is mixed to various degrees with the processed ejecta (equal to zero in our Scenario I).

From those quantities, only the second can be evaluated, in principle, from stellar nucleosynthesis calculations; the other two can only be guessed at, or constrained, by a combination of theory and observations.

Since we lack the required input for item $(b)^{7}$ at present we had to make some assumptions here. We assumed then that the amount of H-processed ejecta is the maximum possible from both potential sources, AGBs or massive stars (Eqs. (12), (13) and Fig. 7). Obviously, any other assumption reducing the

\footnotetext{
7 As mentioned in Sect. 3, the yields of massive AGB stars have been computed recently by several groups in the context of the selfenrichment hypothesis. Nevertheless, the predictions remain highly uncertain (mainly because they depend on the modelling of complex physical mechanisms such as HBB, third dredge-up, convection, mass loss, rotation etc) and none of the present models has properly accounted for the $\mathrm{O}-\mathrm{Na}$ and $\mathrm{Mg}-\mathrm{Al}$ anticorrelations yet. On the other hand, models of rotating massive stars with the appropriate initial composition will soon be available (Decressin et al. 2006).
} 
amount of the processed ejecta would result in IMFs that are even flatter than those found in Sect. 4 (all other things being kept the same). As already discussed in Sect. 4.2, a smaller amount of H-processed ejecta than assumed here is much more plausible physically for both AGB stars and massive stars.

We also made two different assumptions about the composition of the ejecta, namely that they are processed to various degrees (Scenario I) or to an extreme degree (Scenario II). Obviously, the latter assumption is utterly implausible, and it was used only to place absolute lower limits to the slope of the polluter IMF. Indeed, it is hard to conceive that the whole envelope of all the polluters (AGBs or massive stars) is processed to such an extreme degree $([\mathrm{O} / \mathrm{Na}]=-1.4)$; this may happen at some point of the evolution of some of the polluters, but it can characterize neither the totality of the envelope evolution nor the whole mass range of the polluters. Thus, the lower horizontal line of Scenario II (middle panel of Fig. 8) is an extreme and unrealistic lower limit while the upper line of Scenario I is more realistic in that respect. Still, observations suggest that it cannot be true, either.

Indeed, Pasquini et al. (2005) report the presence of $\mathrm{Li}$ in 9 turn-off stars of the intermediate metallicity globular cluster NGC $6752([\mathrm{Fe} / \mathrm{H}]=-1.4)$, a cluster that displays the anticorrelation of $\mathrm{O}$ vs. $\mathrm{Na}$. The $\mathrm{Li}$ abundance in NGC 6752 correlates with the one of $\mathrm{O}$ and anticorrelates with those of $\mathrm{Na}$ and $\mathrm{N}$. The maximum $\mathrm{Li}$ abundance corresponds to stars with $[\mathrm{O} / \mathrm{Fe}] \sim 0.4$, i.e. to stars formed from unprocessed material with a composition similar to the one of field stars. It is no surprise then that the abundance of $\mathrm{Li}$ in those stars $(\log (\mathrm{Li} / \mathrm{H}) \sim-9.6)$ is at the level of the so-called "Spite plateau" observed in field halo stars (e.g., Charbonnel \& Primas 2005). On the other hand, the lowest detected $\mathrm{Li}$ values are about 3 times lower and are found in stars with very low $[\mathrm{O} / \mathrm{Na}] \sim-1.1$ in NGC 6752 .

The presence of so much Li (only within a factor of three below the plateau level) in stars of modified composition in NGC 6752 clearly is at odds with our Scenario I: if such stars are made exclusively from the H-processed ejecta of some class of polluters (as assumed in our Scenario I), then no Li should be present there, in view of the nuclear fragility of that element. And even if one assumes that some fresh Li can be produced in H-burning (and ejected before subsequent destruction) through the Cameron-Fowler (1971) mechanism, it is utterely implausible that the abundance of this new Li is almost as high as the "Spite plateau". The most natural way to explain the presence of $\mathrm{Li}$ in the O-depleted stars of NGC 6752 is to assume that those stars were formed from a mixture of nuclearly processed material with material of normal composition, as in our Scenario II (Prantzos \& Charbonnel, in preparation).

Finally, in both Scenarios I and II we assumed that, while the original IMF contained stars in the full mass range of 0.1 to $100 M_{\odot}$, the ejecta of the polluters only formed stars in the 0.1-0.8 $M_{\odot}$ range, i.e. still alive today. This assumption has no physical justification and was only made in order to maximize the ratio of ejecta to live stars, in the same spirit as the other assumptions discussed in this section. Assuming that the ejecta formed stars in the full $0.1-100 M_{\odot}$ range would obviously lead to flatter IMFs in order to satisfy the observational requirements ${ }^{8}$.

\footnotetext{
8 Assuming that the second ("polluted") generation of stars contained its own massive and fast-evolving "polluters" (the ejecta of which would give rise to a third generation, and so on) would make the whole problem much more complex, and it would invalidate the analysis made here.
}

We conclude then that both our Scenarios I and II are grossly underestimating "reality" in the framework of the selfenrichment hypothesis for globular clusters. A realistic scenario should invoke both ejecta with varying composition (as in Scenario I, more realistic on nucleosynthesis grounds) and mixture of those ejecta with pristine gas (as in Scenario II, to account for Li observations). This implies that the horizontal "observational" lines in Fig. 8 (middle panel) should be replaced by a line lying above the one of Scenario I. Also, a realistic scenario should consider an amount of ejecta considerably lower than the (deliberate) overestimate we made in Eqs. (12) and (13); consequently, the curves of Fig. 8 (middle panel) should shift downwards, as discussed in Sect. 4.3. Overall the slope $X$ of the polluter IMF in a realistic scenario should be much flatter than derived in Sect. 4.3 and certainly much lower than 0 in case the polluters are AGB stars. In other terms, an extremely flat IMF should be necessary to satisfy the constraints imposed by the abundance distribution of Fig. 2.

In such a case, by extrapolating the trends obtained in the lower panel of Fig. 8 (towards the left) one sees that:

- if the polluters are AGB stars, the mass of the cluster today should be completely dominated by residues (by a factor of several);

- in contrast, if the polluters are massive stars, the total mass of the residues today would be much lower than the mass of live stars in the cluster.

With these implications in mind, we now take a closer look at the self-enrichment scenario, for both invoked classes of polluters.

\subsection{Polluters: AGBs vs. WMS}

\subsubsection{AGBs?}

According to a commonly held idea, the peculiar chemical composition of GC stars stems from pollution by the ejecta of AGB stars, massive enough (4-9 $\left.M_{\odot}\right)$ to develop HBB in their envelopes. Such stars evolve in time scales of $\sim 20$ to $150 \mathrm{Myr}$ and release their ejecta at velocities $v<30 \mathrm{~km} \mathrm{~s}^{-1}$ (e.g., Loup et al. 1993), lower than the escape velocity from the cluster; the ejecta are trapped in the cluster and form new stars, either directly (our Scenario I) or indirectly, after mixing with pristine material to various degrees (our Scenario II, which accounts for the Li observations, as argued in Sect. 5.1).

In our opinion, this idea suffers from several drawbacks (in addition to the problematic nucleosynthesis predictions of the current models discussed in Sect. 3). In particular, it gives no satisfactory answer to the roles of stars that are more massive $\left(M>9 M_{\odot}\right)$ and less massive $\left(M<4 M_{\odot}\right)$ than the presumed polluters.

1) Stars more massive than $9 M_{\odot}$ evolve on time scales lower than $20 \mathrm{Myr}$ and are quite numerous (since a flat IMF is required in all cases to account for the observed abundance distribution, as argued in this work). They release material processed through various nuclear burning stages, both through their winds (slow in the main sequence, quite fast in the Wolf-Rayet stage) and through the final stellar explosion as supernovae. A commonly given answer to the fate of their ejecta is that the fast-moving supernova material $\left(v>400 \mathrm{~km} \mathrm{~s}^{-1}\right.$ even for the innermost SN regions) escapes the system and leaves no trace behind. On its way, it sweeps up the previously released wind ejecta, but also any ambient gas of pristine composition. Consequently, no original $\mathrm{Li}$ rich gas is left behind to subsequently get mixed with the AGB ejecta. The second (polluted) stellar generation should 
then be formed exclusively with AGB ejecta, which implies that AGBs should produce their own $\mathrm{Li}$ at a level close to the Spite plateau. This is an extremely strong constraint on the nucleosynthesis of AGB stars.

Alternatively, one may argue that an insignificant amount of nuclearly processed ejecta escapes the supernovae, the vast majority being trapped within a black hole. This is an utterly implausible scenario, since neutron stars and pulsars are common in the Milky Way, and there is no reason for massive stars in GCs not to produce such residues.

Finally, one might also invoke a peculiar IMF of the first stellar generation in GCs, which lacks massive stars altogether. Such an ad hoc assumption, however, is not supported by any theoretical arguments or numerical simulation results.

2) Stars in the mass range $2-4 M_{\odot}$ evolve on timescales of 150 to $1000 \mathrm{Myr}$ and release almost as much mass as those of 4-9 $M_{\odot}$ (depending on the assumed IMF: for $X=0.15$ we obtain $E(2-4)=0.35 E(4-9))$. Even if they do not develop $\mathrm{HBB}$ in their envelopes, they go through the third dredge-up phase, which brings He-burning products (in particular C) and s-elements to the surface, slowly releasing them to the ISM. If new stars are formed from these ejecta, $\mathrm{C}$ and s-element excesses should be observed in GC stars, which is not the case. One then has to assume that the ejecta of 2 to $4 M_{\odot}$ form no new stars, why those of their slightly more massive sisters manage to do it very efficiently. Perhaps, on time scales $>100 \mathrm{Myr}$, tidal interactions of the GCs with the Galactic potential are able to remove most of the cluster gas (as well as part of its stars); still, some ejecta should be left behind to form stars with $\mathrm{C}$ and s-elements excess. Until a consistent dynamical scenario is developed to convincingly account for that point, we consider it to be one of the drawbacks of the AGB scenario for the pollution of GCs.

\subsubsection{WMS?}

The idea that WMS may be at the origin of some anomalies in the composition of GCs has been recently suggested by Norris (2004) and Maeder \& Meynet (2006), in order to explain the blue main sequence of the cluster $\omega$ Centauri. The high helium content of the stars of that sequence could originate in WMS, producing a large helium/metal ratio. Omega Cen is, however, a peculiar case, showing a dispersion in its heavy metal content, unlike any other GC. If the polluters of GCs are indeed the winds of massive stars, the scenario could run along the following lines.

The winds of those stars, rich in H-burning products (especially if the stars are rotating), are slowly released in the ambient ISM and they push it, opening circumstellar cavities. They are finally mixed with pristine gas, still left over in the system after the formation of the first stellar generation. By reaching the walls of these cavities, the shock waves of the subsequent SN explosions induce the formation of new stars from this gas of mixed composition. The hot SN ejecta inside the shock waves are expanding mainly along the cavities opened up previously by the massive star winds, and they finally find their way out of the cluster, escaping the system altogether. Thus, the stars we now observe in the cluster either have their original composition, or a composition resulting from the mixture of pristine gas with WMS. On longer timescales ( $>20 \mathrm{Myr}$ ), the wind ejecta of lower-mass stars $\left(M<10 M_{\odot}\right)$ that do not explode are slowly released in the cluster. However, they never form new stars, since there is no external agent to trigger condensation of that gas and to induce star formation. As discussed before, tidal interactions ultimately strip that gas off the cluster.
The advantages of the WMS scenario are that i) it may "naturally" account for the formation of the second stellar generation with mixed composition, which is triggered by the SN shock waves; and ii) it may also explain why no trace of the He-burning products of the third dredge-up (which certainly occurs in lowmass AGBs) is seen today in GC stars. These products, along with those of $\mathrm{HBB}$ in more massive AGBs, are slowly released in a rarefied medium (since almost all of the gas of the system has been swept up or condensed previously, after the passage of the shock waves); as a result, they never condense to form new stars (for lack of triggering agent) and they are ultimately removed from the cluster through tidal interactions.

Of course, this novel scenario also has its potential drawbacks. It assumes that WMS mix thoroughly with pristine gas and that the subsequent shock wave triggers efficient star formation in the mixture, while the SN ejecta escape the system. One may, in fact, imagine the opposite situation where the shock waves disperse the gas or the SN ejecta mix with that gas (at least partially). At the current stage of our knowledge, both alternatives are possible.

The WMS scenario sketched here is, admittedly, at a very qualitative level (but no more than the scenario involving AGBs). It should be substantiated at least by nucleosynthesis calculations demonstrating that WMS can indeed provide the chemical composition required to explain the observations of abundances in GCs; such calculations have already been performed and they will be presented elsewhere (Decressin et al. 2006). We feel, however, that the WMS scenario opens very interesting perspectives for the understanding of the self-enrichment of GCs.

\section{Summary}

In this work we have studied the constraints on the IMF of stars responsible for the observed abundance anomalies in GCs, in the framework of the so-called self-enrichment scenario. At present, there is neither a unique nor a well-defined, nor even a self-consistent self-enrichment scenario for GCs. The parameter space is quite large and involves (at least) the IMF (mass limits and shape) of the polluter stars, the amount and composition of their ejecta, as well as the amount of unprocessed gas, which is mixed to various degrees with these ejecta to form the observed contaminated low-mass stars.

In view of the many uncertainties related to the selfenrichment scenario, we explore several possibilities in details, but our study is far from exhaustive in that respect (Sect. 3). In particular, we explore two different types of self-enrichment scenarios, differing in the composition of the polluter ejecta, i.e. the degree to which the ejecta are contaminated by H-burning products. In Scenario I, second generation stars are formed exclusively from ejecta processed at various degrees, while in Scenario II they are formed from extremely processed ejecta mixed to various degrees with pristine material. Also, we explore two different possibilities for the polluters, namely AGB stars (4-9 $M_{\odot}$ ) and WMS (>10 $\left.M_{\odot}\right)$, since both of them can, in principle, release H-burning products in the ISM at slow velocities. In each case we assume that the amount of ejecta is as large as possible, in order to constrain the polluter IMF from one side.

We adopt a composite IMF (Sect. 4.1) with an observationally-derived part in the mass range $0.1-0.8 M_{\odot}$ (from PdM00) and a power-law for higher masses (i.e. for stars that are extinguished today); our aim was to constrain the slope $X$ of the power-law part of the IMF. For a concrete illustration of our method, we chose NGC 2808, the GC for which the largest sample of $\mathrm{O}$ and $\mathrm{Na}$ abundances is presently 
available. We derived the abundance distribution of $\mathrm{O} / \mathrm{Na}$ in that cluster (Sect. 2) and find that $\sim 30 \%$ of its stars have a pristine composition, while the remaining $70 \%$ are contaminated to various degrees by H-burning products.

We then show how this contamination can be obtained quantitatively in the framework of our two self-enrichment scenarios and under the different assumptions made about the mass range of the polluters (AGBs vs. WMS). We find that, in all cases, the IMF of the polluters has to be flatter than the standard presentday IMF (i.e. with the Salpeter slope $X=1.35$ ). The trend is more pronounced in Scenario I than in Scenario II, and also more pronounced in the case of AGBs than in the case of WMS (Sect. 4.3). However, due the many uncertainties involved, it is impossible to make a realistic quantitative prediction about the upper limit of the slope $X$; in fact, we argue in Sect. 5.1 that a realistic scenario would lead to an even much flatter IMF than those obtained in this work.

An IMF with $X<-0.4$ (i.e. much flatter than obtained here) was found in the work of Smith \& Norris (1982) for NGC 6752 and 47 Tuc, in order to explain the observed $C$ depletion in the framework of the self-enrichment scenario. By using absolute abundances (and not only abundance ratios, as in our work) they were also able to place an upper limit of $11 M_{\odot}$ to the IMF of the polluters. Clearly, such work should be repeated with updated stellar yields and a wide basis of spectroscopic data on as many elements as possible, in order to constrain the polluter IMF and even the validity of the various self-enrichment scenarios.

We find that the amount of stellar residues (both by mass and by number) present in the GC, is sensite to the slope of the adopted IMF and to the mass range of the polluters, thereby providing another potential discriminating test between the various possibilities. We illustrate this (Sect. 4.4) by comparing our results to the number ratio $(\sim 1)$ of white dwarfs to low-mass stars inferred in the cluster M4. We find that our results fall short of producing such a large ratio, the discrepancy being more important in the case of WMS than in the case of AGBs. In fact, taken at face value, our results might be interpreted as a complete failure of the self-enrichment scenario (and as an argument for the internal evolution scenario). We argue, however, that this "failure" may be attributed to the extreme assumptions adopted here and that more realistic assumptions would produce results closer to the observations.

Finally, in Sect. 5 we discuss the various assumptions made in our study. In particular, we comment on some problems associated (in our opinion) with AGBs, the most frequently-invoked polluters of GCs (Sect. 5.2.1). We also develop for the first time a possible scenario for the pollution of GCs by WMS in some detail, and also invoke its own potential problems (Sect. 5.2.2). We conclude that WMS offer an appealing alternative to AGBs as polluter candidates of GCs.

Overall, our study shows that the self-enrichment scenario of GCs can be quantitatively tested, through progress in observations (abundance distributions for several elements, number counts of white dwarfs and low mass stars, dynamical determination of the baryonic mass of a given cluster), and theory (improved understanding of the amount and composition of the ejecta of the various candidate polluters).

Acknowledgements. We are indebted to E. Carretta for providing us with the NGC 2808 data prior to publication. We wish to warmly thank G. Meynet and G. Wallerstein for enlightening comments and a careful reading of the manuscript, as well as T. Decressin, G. de Marchi, G. Meylan, and P. Molaro for useful discussions. We thank the anonymous referee for constructive comments.

\section{References}

Adams, F. C., \& Fatuzzo, M. 1996, ApJ, 464, 256

Armosky, B. J., Sneden, C., Langer, G. E., \& Kraft, R. P. 1994, AJ, 108, 1364

Bedin, L. R., Piotto, G., Zoccali, M., et al. 2000, A\&A, 363, 159

Brown, J. H., Burkert, A., \& Truran, J. W. 1991, ApJ, 376, 115

Cameron, A. G. W., \& Fowler, W. A. 1971, ApJ, 164, 111

Carretta, E., Bragaglia, A., Cacciari, C., \& Rossetti, E. 2003, A\&A, 410, 143

Carretta, E., Bragaglia, A., \& Cacciari, C. 2004, ApJ, 610, L25

Carretta, E. 2006, AJ, 131, 1766

Carretta, E., Bragaglia, A., Gratton, R. G., et al. 2006, A\&A, 450, 523

Castellani, V., Iannicola, G., Bono, G., et al. 2006, A\&A, 446, 569

Cayrel, R. 1986, A\&A 168, 81

Charbonnel, C. 2005, From lithium to uranium: Element tracers of early cosmic evolution (Cambridge Univ. Press), ed. V. Hill, P. François, \& F. Primas, IAU Symp., 228, 347

Charbonnel, C., \& Primas, F. 2005, A\&A, 442, 961

Cohen, J. G. 1978, ApJ, 223, 487

Cottrell, P. L., \& Da Costa, G. S. 1981, ApJ, 245, L79

D’Antona, F., Bellazzini, M., Caloi, V., et al. 2005, ApJ, 631, 868

D'Antona, F., \& Caloi, V. 2004, ApJ, 611, 871

D'Antona, F., Caloi, V., Montalban, J., Ventura, P., \& Gratton, R. 2002, A\&A, 395, 69

D’Antona, F., Gratton, R., \& Chieffi, A. 1983, Mem. Soc. Astron. It., 54, 173

Decressin, T., \& Charbonnel, C. 2005, From lithium to uranium: Element tracers of early cosmic evolution (Cambridge Univ. Press), ed. V. Hill, P. François, \& F. Primas, IAU Symp., 228, 395

Decressin, T., Meynet, G., Charbonnel, C., Prantzos, N., \& Ekström, S. 2006, A\&A, submitted

De Marchi, G., Paresce, F., Straniero, O., \& Prada Moroni, P. G. 2004, A\&A, 415,971

Denissenkov, P. A., \& Denissenkova, S. N. 1990, SvA Lett., 16, 275

Denissenkov, P. A., \& Herwig, F. 2003, ApJ, 590, 99

Drake, J. J., Smith, V. V., \& Suntzeff, N. B. 1992, ApJ, 395, L95

Dickens, R. J., Croke, B. F. W., Cannon, R. D., \& Bell, R. A. 1991, Nature, 351, 212

Elmegreen, B. G. 1999, ApJ, 515, 323

Fall, S. M., \& Rees, M. J. 1985, ApJ, 298, 18

Fenner, Y., Campbell, S., Karakas, A. I., Lattanzio, J. C., \& Gibson, B. K. 2004, MNRAS, 353, 789

Gratton, R., Bonifacio, P., Bragaglia, A., et al. 2001, A\&A, 369, 87

Giersz, M. 2001, MNRAS, 324, 218

Goriely, S., \& Siess, L. 2005, From lithium to uranium: Element tracers of early cosmic evolution (Cambridge Univ. Press), ed. V. Hill, P. François, \& F. Primas, IAU Symp., 228, 451

Gratton, R., Sneden, C., \& Carretta, E. 2004, ARA\&A, 42, 385

Grundahl, F., Briley, M., Nissen, P. E., \& Feltzing, S. 2002, A\&A, 385, L14

Harris, W. E., \& Pudritz, R. E. 1994, ApJ, 429, 177

Herwig, F. 2004a, ApJ, 605, 425

Herwig, F. 2004b, ApJS, 155, 651

Iben, I. 1976, ApJ, 208, 165

Iben, I., \& Tutukov, A. 1984, ApJ, 284, 719

Ivans, I. I., Sneden, C., Kraft, R. P., et al. 1999, AJ, 118, 1273

Ivans, I. I., Kraft, R. P., Sneden, C., et al. 2001, AJ, 122, 1438

James, G., François, P., Bonifacio, P., et al. 2004a, A\&A, 414, 1071

James, G., François, P., Bonifacio, P., et al. 2004b, A\&A, 427, 825

Joshi, K. J., Nave, C. P., \& Rasio, F. A. 2001, ApJ, 550, 691

Karakas, A. I., \& Lattanzio, J. C. 2003, PASA, 20, 279

Kraft, R. P. 1994, PASP, 106, 553

Kroupa, P. 2002, Science, 295, 82

Langer, G. E., Hoffman, R., \& Sneden, C. 1993, PASP, 105, 301

Lee, Y. W., Joo, S. J., Han, S. I., et al. 2005, ApJ, 621, L57

Loup, C., Forveille, T., Omont, A., \& Paul, J. F. 1993, A\&AS, 99, 291

Maeder, A., \& Meynet, G. 2006, A\&A, 448, L37

Michaud, G., Richard, O., \& Richer, J. 2004, Mem.S.A.It., 75, 339

Norris, J. 1981, ApJ, 612, L25

Norris, J. E., Cottrell, P. L., Freeman, K. C., \& Da Costa, G. S. 1981, ApJ, 244, 205

Paresce, F., \& De Marchi, G. 2000, ApJ, 534, 870 (PdM00)

Parmentier, G. 2004, MNRAS, 351, 585

Parmentier, G., Jehin, E., Magain, P., et al. 1999, A\&A, 352, 138

Pasquini, L., Bonifacio, P., Molaro, P., et al. 2005, A\&A, 441, 549

Peterson, R. C. 1980, ApJ, 237, L87

Pulone, L., De Marchi, G., Paresce, F., \& Allard, F. 1998, ApJ, 492, L41

Pilachowski, C. A. 1989, in The abundance spread within globular clusters, ed. G. Cayrel de Strobel, M. Spite, \& T. L. Evans, 1

Ramirez, S. V., \& Cohen, J. G. 2002, AJ, 123, 3277

Ramirez, S. V., \& Cohen, J. G. 2003, AJ, 125, 224 
Richard, O., Michaud, G., Richer, J., et al. 2002, ApJ, 568, 979

Richer, H., Brewer, J., Fahlman, G., et al. 2002, ApJ, 574, L151 (RBF02)

Rubenstein, E., \& Bailyn, C. 1999, ApJ, 513, L33

Salpeter, E. E. 1955, ApJ, 121, 161S

Shetrone, M. D. 1996, AJ, 112, 1517

Smith, G. H., \& Norris, J. 1982, ApJ, 254, 594

Smith, G. H., Shetrone, M. D., Bell, R., Churchill, C. W., \& Briley, M. M. 1996, AJ, 112, 1511

Smith, V. V., Cunha, K., Ivans, I. I., et al. 2005, ApJ, 633, 392

Sneden, C. 2005, From lithium to uranium: Element tracers of early cosmic evolution (Cambridge Univ. Press), ed. V. Hill, P. François, \& F. Primas, IAU Symp., 228, 337

Sneden, C., Gratton, R. G., \& Crocker, D. A. 1991, A\&A, 246, 354

Sneden, C., Kraft, R. P., Guhathakurta, P., Peterson, R. C., \& Fullbright, J. P. 2004, AJ, 127, 2162
Sosin, C., Dorman, B., Djorgovski, G., et al. 1997, ApJ, 480, L35

Spite, M., Cayrel, R., \& Plez, B. 2005, A\&A, 430, 655

Stahler, S., \& Palla, F. 2005, The formation of stars (J. Wiley)

Thoul, A., Jorissen, A., Goriely, S., et al. 2002, A\&A, 383, 491

Truran, J. 1988, The Impact of Very High S/N Spectroscopy on Stellar Physics, IAU Symp., 132, 577

Truran, J. W., Brown, J., \& Burkert, A. 1991, The formation and Evolution of Star Clusters, ASP Conf. Ser., 13, 78

Ventura, P., \& D'Antona, F. 2005a, A\&A, 431, 279

Ventura, P., \& D'Antona, F. 2005b, A\&A, 439, 1075

Ventura, P., \& D'Antona, F. 2005c, ApJ, 635, L149

Ventura, P., D’Antona, F., Mazzitelli, I., \& Gratton, R. 2001, ApJ, 550, L65

Ventura, P., D’Antona, F., \& Mazzitelli, I. 2002, A\&A, 393, 215

Zinnecker, H. 1984, MNRAS, 210, 43 